\title{
AUFSÄTZE
}

\author{
Ulrich Roos/Timo Seidl
}

\section{Im »Südwesten« nichts Neues? Eine Analyse der deutschen Namibiapolitik als Beitrag zur Rekonstruktion der außen- politischen Identität des deutschen Nationalstaates}

Der Beitrag widmet sich der Frage, welche grundlegenden Handlungsregeln die deutsche Namibiapolitik anleiten und welche Rückschlüsse sich daraus für die Diskussion um das Verhältnis von Interessen und Werten in der deutschen Außenpolitik ziehen lassen. Vor dem Hintergrund der für deutsche Außenpolitik einzigartigen Gegenwärtigkeit der kolonialen Vergangenheit interessieren wir uns dafür, was sich aus der Art und Weise des Umgangs der Bundesregierung mit dieser Vergangenheit über die gegenwärtige Beschaffenheit der (außen-)politischen Kultur des deutschen Nationalstaates lernen lässt. Dabei greifen wir auf Methoden der rekonstruktiven Weltpolitikforschung in Anlehnung an die Grounded Theory in der Lesart von Anselm Strauss zurück und gelangen zu der These, dass die deutsche Namibiapolitik nicht als Ausdruck einer zivilmächtigen Orientierung verständlich gemacht werden kann. Vielmehr bestätigt diese, dass die grundlegende Eigenschaft deutscher Außenpolitik in der kontinuierlichen Priorität deutscher Interessen besteht, wenngleich diese Interessen durchaus mehrdimensional definiert und flexibel verfolgt werden.

Schlagworte: Deutsche Außenpolitik, deutsch-namibische Beziehungen, Rekonstruktive Weltpolitikforschung, Erinnerungspolitik, Kolonialismus

»Recognition of the inherent dignity and of the equal and inalienable rights of all members of the human family is indispensable for freedom, justice and peace« (Präambel der Verfassung der Republik Namibia).

»Im Bewußtsein seiner Verantwortung vor Gott und den Menschen, von dem Willen beseelt, als gleichberechtigtes Glied in einem vereinten Europa dem Frieden der Welt zu dienen, hat sich das Deutsche Volk kraft seiner verfas- 
sungsgebenden Gewalt dieses Grundgesetz gegeben « (Präambel des Grundgesetzes der Bundesrepublik Deutschland).

\section{Einleitung}

Die Frage, inwieweit deutsche Außenpolitik dem normativen Idealtyp einer Zivilmacht (Maull 1992) (noch immer) entspricht, ist heute - angesichts der allerorten skizzierten weltpolitischen Verwerfungen und der allseits diagnostizierten Veränderungen deutscher Außenpolitik (Baumann 2006; Hellmann et al. 2008; Maull 2011) - aktueller denn je. ${ }^{1}$ Im Kern der gesellschaftlichen und politikwissenschaftlichen Debatte zur Entwicklung der deutschen Außenpolitik standen und stehen dabei die Fragen, ob sich in deren Wertegefüge, also in der Priorisierung normativ wie analytisch unterscheidbarer Werte, 1.) Veränderungen ergeben oder nicht, 2.) welcher Art mögliche Veränderungen sind, 3.) wie sie sich erklären lassen sowie 4.) welches Gesamtbild dies, mit Blick auf die Prioritäten und die im Raum stehende Frage nach der Intensität des Wandels der zivilmächtigen Identität deutscher AuBenpolitik, ergibt.

Um einen Beitrag zur Beantwortung dieser Fragen zu leisten, wollen wir jedoch nicht (noch einmal) eine der großen Arenen deutscher Außenpolitik beforschen, sondern wenden uns stattdessen einem ihrer bislang weitgehend vernachlässigten Bereiche zu: der deutschen Namibiapolitik. Abgesehen von deren Bedeutung für ein besseres Verständnis deutscher Süd-, Afrika- und Entwicklungspolitik, ist es vor allem die Gegenwärtigkeit der kolonialen Vergangenheit, welche die deutsche Namibiapolitik zu einem besonderen und besonders interessanten Fall deutscher Außenpolitik macht. Nicht nur, dass wir es mit dem einzig(artig)en Beispiel deutscher Außenpolitik gegenüber einer ehemaligen deutschen Siedlungskolonie zu tun haben; diese Außenpolitik findet auch vor dem Hintergrund einer nie wirklich erfolgten gesellschaftspolitischen Auseinandersetzung mit der deutschen kolonialen Vergangenheit und darüber hinaus im Schatten eines Völkermordvorwurfs statt, über

1 Herzlich bedanken möchten wir uns an dieser Stelle bei unseren Gesprächs- und Interviewpartnern in Deutschland und Namibia für die Bereitschaft, ihre Perspektiven auf unseren Untersuchungsgegenstand mit uns zu teilen, bei den TeilnehmerInnen des Augsburger Forschungskolloquiums, in welchem eine frühere Version des Papiers kontrovers und konstruktiv diskutiert wurde, bei der Universität Augsburg, die unsere Namibiareise großzügig bezuschusst hat, bei den zahlreichen Studierenden, die im Rahmen von Lehrveranstaltungen an der Entwicklung unserer Ideen maßgeblichen Anteil hatten, bei Ulrich Franke, Moritz Laurer und Charlotte Rungius für ihre stetige und stets produktive Begleitung unseres Forschungsvorhabens sowie bei den anonymen Gutachtern für ihre ebenso kenntnis- wie hilfreichen Anmerkungen. 
dessen Berechtigung unter Fachhistorikern im Grunde kein Zweifel besteht (siehe Abschnitt 3).

Es ist diese geteilte Kolonialgeschichte, welche die Beziehungen zwischen der Bundesrepublik Deutschland und der Republik Namibia wie ein leises, aber doch vernehmbares Hintergrundrauschen begleitet und belastet und welche die deutsche Namibiapolitik zu einem Fall macht, von dem wir glauben, dass er zu einem besseren Verständnis der grundlegenden Eigenschaften deutscher Außenpolitik beitragen kann. War die deutsche Namibiapolitik ihrem bisher einzigen, genuin politikwissenschaftlichen und $u$. a. am Konzept der Zivilmacht orientierten Erklärungsangebot (Engert 2009: 285-286) ein »theoretische[s] puzzle« geblieben (Engert 2009: 295), so ist es unser Anliegen, dieses Rätsel aufzunehmen und einen Beitrag zu seiner Lösung in Form einer Theorie der grundlegenden Eigenschaften der jüngeren deutschen Namibiapolitik zu leisten. Vor diesem Hintergrund stellt sich uns die Forschungsfrage, welche grundlegenden Handlungsregeln die deutsche Namibiapolitik anleiten und wie sich diese Handlungsregeln zu den an anderer Stelle formulierten Thesen einer Theorie deutscher Außenpolitik (Roos 2010; Laurer/ Seidl 2014) verhalten. Es sollen also nicht zuletzt frühere Thesen und Theorien der Verfasser bezüglich der Entwicklung deutscher Außenpolitik erneut auf den Prüfstand gestellt und gegebenenfalls modifiziert werden.

Hierfür werden wir zunächst die dieser Fragestellung zugrundeliegenden ontologischen und epistemologischen Prämissen sowie die für ihre Bearbeitung ausgewählte methodische Vorgehensweise explizieren (Abschnitt 2). Da die Auseinandersetzung mit dieser Fragestellung im Grenzgebiet zwischen Politik- und Geschichtswissenschaft erfolgte, möchten wir unserem politikwissenschaftlichen Antwortversuch unsere Rekonstruktion wesentlicher Elemente der Geschichte der deutsch-namibischen Beziehungen als fallbezogenes Kontextwissen voranstellen (Abschnitt 3). Den Hauptteil der Arbeit bildet die Darstellung unserer zentralen Befunde und ihrer Implikationen für unsere eigenen, aber auch für im Forschungsstand vertretene Thesen bezüglich der Beschaffenheit deutscher Außenpolitik (Abschnitt 4). Abschließend formulieren wir drei politikberatende Argumente, wieso deutsche (Außen-)Politik die koloniale Vergangenheit ernster nehmen sollte als bisher (Abschnitt 5).

\section{Formaltheoretische Prämissen und methodische Vorgehensweise}

Jede Forschungsfrage gründet auf ontologischen Prämissen und orientiert sich an epistemologischen Überzeugungen, die gemeinsam die Auswahl geeignet erscheinender Methoden zur Bearbeitung des aufgeworfenen Problems bestimmen. Erst 
indem diese methodologischen Überzeugungen und Entscheidungen wenigstens kurz skizziert werden, kann die Forschungsidee von den LeserInnen angemessen verstanden und damit auch pointiert kritisiert werden.

\subsection{Ontologische Prämissen}

Der in der Forschungsfrage zentrale Begriff der Handlungsregel geht auf den klassischen Pragmatisten Charles Sanders Peirce zurück. Dieser ging davon aus, dass die Überzeugungen der Menschen als Handlungsregeln zu denken seien, Akteure also aufgrund ihrer Überzeugungen handeln. Dabei begriff er Überzeugungen als Resultat von Interaktion, von Praxis und Erfahrung. Handlungsregeln können an der Praxis scheitern, Menschen und Kollektive in Krisen geraten, was eine Veränderung bisheriger Handlungsregeln notwendig macht. Menschen können also lernen (ob bewusst oder unbewusst ist hierbei irrelevant) und ihre Handlungsregeln sind Resultat eines komplexen prozessualen, lebendigen Gewebes von Zweifel und Glaube, von Krise und Routine, von Kontinuität und Wandel. Die Menge aller Handlungsregeln eines Akteurs begreifen wir als dessen Identität.

John Dewey führte die Figur der »Strukturen kollektiven Handelns « ein, um den Zusammenhang zwischen menschlichen und kollektiven Akteuren zu modellieren (Dewey 1991: 190). Dabei bekleiden einzelne Menschen innerhalb einer bestimmten Struktur kollektiven Handelns, zum Beispiel der Bundesregierung oder dem Auswärtigen Amt, bestimmte Strukturpositionen. Diese Strukturpositionen sind untereinander verwoben, regeln die Kompetenzen der Beteiligten und geben einen Raum möglicher - weil denkbarer und praktizierbarer - Handlungsregeln vor. Dieser Raum möglicher Handlungen ist von einzelnen Personen nicht beliebig gestaltbar, sondern wird zuvorderst sozial verhandelt. Denn alle Möglichkeitsräume einer jeden Strukturposition einer Struktur kollektiven Handelns sind an einen gemeinsamen Bestand von Handlungsregeln gebunden, der die Identität der Struktur konstituiert und ihr Handeln bestimmt. Praxis, Irritation, Zweifel und Erfahrung einzelner Menschen können diesen kollektiven Möglichkeitsraum verändern, können die Kompetenzen einzelner Strukturpositionen in Bewegung setzen, doch über die Ausgestaltung der Struktur kollektiven Handelns entscheiden in erster Linie nicht Einzelne, sondern das Kollektiv, ob bewusst oder nicht (Franke/Roos 2010; Roos 2015).

\subsection{Epistemologisch-methodologische Perspektive}

Unser Ansatz basiert auf der Annahme, dass die Handlungsregeln der (kollektiven) Akteure, die wir rekonstruieren wollen, durch die Analyse protokollierter Spuren 
von (Sprech-)Handlungen dechiffriert werden können (Oevermann 1991: 295). Dabei stellen sowohl die Handlungen der uns interessierenden Akteure (hier der Bundesregierung) als auch die Reaktionen ihrer Umwelt Material für uns dar, da wir in Anlehnung an die pragmatistische Maxime von Peirce (Peirce 1998: 338) davon ausgehen, dass die Identität eines Gegenstands aus allen Wirkungen besteht, die dieser (potenziell) zeitigt. Wer die Identität der deutschen Namibiapolitik rekonstruieren möchte, muss folglich neben den (Sprech-)Handlungen der Bundesregierung auch die diesbezüglichen Reaktionen berücksichtigen, oder - was einen methodisch zu reflektierenden Sonderfall darstellt - solche Fremdbeschreibungen durch das Führen von Interviews auslösen.

Dabei begreifen wir die von uns generierte Theorie der Identität deutscher Namibiapolitik als stets fallibel. Wir behaupten keine ewige Wahrheit auszusprechen, was aufgrund der Lebendigkeit des Sozialen und Politischen kein haltbarer Standpunkt wäre, sondern eine zum jetzigen Zeitpunkt plausibel erscheinende Theorie zu formulieren. Das von uns generierte »Wissen« will dabei andere Theorien nicht tilgen und verdrängen, sondern stellt sich neben andere Lesarten und überlässt es den FachkollegInnen, den mit diesem Fall vertrauten PraktikerInnen und der größeren Gesellschaft die Argumente zu prüfen und die Qualität der Theorie zu bewerten. Nicht das letzte, sondern ein besonders plausibles Wort soll hier zur Frage der deutschen Namibiapolitik gesprochen werden.

\subsection{Methodische Vorgehensweise}

Wie frühere Arbeiten orientiert sich auch diese Studie an Vorschlägen methodischen Arbeitens, die an anderer Stelle unter der Bezeichnung »Methoden rekonstruktiver (Weltpolitik-)Forschung « detailliert erörtert wurden (Franke/Roos 2010; Franke/ Roos 2013; Roos 2013). Zentral für unser Vorgehen war dabei 1.) das theoretische Kodieren in Anlehnung an Anselm Strauss, also das systematische Fahnden nach Eigenschaften des Untersuchungsgegenstands und nach zwischen diesen Eigenschaften bestehenden Zusammenhängen, die in Form von Hypothesen formuliert werden und gemeinsam eine auf den Gegenstand bezogene Theorie ergeben, sowie 2.) das Theoretical-sampling-Konzept von Strauss, also die Idee, nicht zu Beginn der Studie darüber zu entscheiden, welche Daten analysiert werden sollen, sondern dies stets von Fall zu Fall neu, nach Kriterien der minimalen und maximalen Kontrastierung, zu entscheiden, um die konzeptionelle Repräsentativität zu steigern, anstatt anzunehmen, eine Repräsentativität der Stichprobe für die Grundgesamtheit durch eine Vorabfestlegung des Datensatzes erreichen zu können. Die Idee ist also, erst im Laufe der Theoriegenese immer besser zu begreifen, welche Aspekte noch 
zu wenig belichtet wurden und welche weiteren Beobachtungen notwendig erscheinen, um die Theorie zu verfeinern und mit Blick auf wenigstens die grundlegenden Eigenschaften des Untersuchungsgegenstands zu vervollständigen (Strauss 1987; Strauss/Corbin 1998; Strübing 2005).

Während des gesamten Prozesses verliefen Datenauswahl, Datenanalyse und Lektüre bereits vorliegender theoretischer Überlegungen Dritter parallel. Auch wenn wir uns als Forscherkollektiv in einem kontinuierlichen Austauschprozess befanden, entschieden wir uns auf Ebene der konkreten Kodierung des Materials zunächst getrennt voneinander zu arbeiten und die jeweiligen Befunde in einem zweiten Schritt zu einer gemeinsamen Theorie zu verbinden. Dabei erkannten wir schnell, dass wir deutsche Namibiapolitik allein vom Schreibtisch aus nicht angemessen verstehen konnten und beschlossen daher, 1.) zusätzlich nach Namibia zu reisen, um unsere Theorie der deutschen Namibiapolitik vor Ort durch Gespräche mit Vertretern der relevanten Interessengruppen zu schärfen, ${ }^{2}$ sowie 2.) das Gespräch mit Heidemarie Wieczorek-Zeul zu suchen, um unsere derart geschärfte Theorie im Sinne des von Strauss als member check bezeichneten Verfahrens zur Diskussion zu stellen. ${ }^{3}$

Im nachfolgenden Befundkapitel werden wir aus der großen Zahl kodierter Sequenzen nur einige wenige auswählen, um unsere Thesen illustrativ zu belegen. Eine solche Belegstrategie bliebe stark angreifbar, stünde sie für sich alleine. Aus diesem Grund sind soweit möglich die Kodierbäume - auch langfristig - im Internet veröffentlicht.

2 Im Zuge eines Forschungsaufenthalts in Namibia führten wir Interviews und Hintergrundgespräche mit Anton von Wietersheim (Mitglied des namibischen Parlaments und ehemaliger Minister für Landwirtschaft), Eberhard Hofmann (Stellvertretender Chefredakteur der deutschsprachigen Allgemeinen Zeitung), Heiner Naumann (Büroleiter der Friedrich-Ebert-Stiftung in Namibia) und Bernd Althusmann (Büroleiter der Konrad-Adenauer-Stiftung in Namibia) sowie mit Angehörigen der Gemeinschaften der Herero und Nama, die wir zum Schutz ihrer Person nicht namentlich nennen wollen. Darüber hinaus interviewten wir einen hochrangigen Repräsentanten der Bundesrepublik Deutschland, dessen Aussagen wir hier in namentlich anonymisierter Form veröffentlichen, nachdem die Presseabteilung des Auswärtigen Amts die im Rahmen des Tonbandinterviews protokollierten Aussagen im Nachhinein nicht freigeben wollte, obgleich Nutzungsabsichten und Forschungskontext im Vorfeld transparent gemacht wurden.

3 Um nicht missverstanden zu werden: Sinn und Zweck des member checks ist es natürlich nicht, die ausgewählten ExpertInnen entscheiden zu lassen, welche Befunde »wahr« sind und welche »unwahr«. Vielmehr liefern solche Interviews und Hintergrundgespräche einerseits zusätzliches Material und ermöglichen andererseits - im Sinne einer Prüfung und Verfeinerung der Theorie - zu »testen«, wie die ExpertInnen und PraktikerInnen auf bestimmte Thesen reagieren. 


\section{Eine kurze Geschichte der deutsch-namibischen Beziehungen}

Die in Abschnitt 2.2 explizierten erkenntnistheoretischen Überzeugungen bestimmen auch den Stellenwert der nachfolgenden Rekonstruktion der deutsch-namibischen Geschichte. Wir behaupten nicht zu wissen, wie »es wirklich gewesen ist«, sondern entwerfen 1.) auf Grundlage der Rekonstruktion des hierzu veröffentlichten geschichtswissenschaftlichen Forschungsstandes, 2.) des selbst gesichteten historischen Materials (wie z. B. Feldtagebücher der deutschen Schutztruppe und Protokolle von Reichstags- und Bundestagsdebatten) sowie 3.) unter Berücksichtigung sämtlicher im Rahmen von Interviews und Gesprächen kommunizierter Theorien Dritter bezüglich vergangener Handlungen unsere Rekonstruktion der historischen Ereignisse.

\subsection{Vom deutschen Schutz- zum südafrikanischen Mandatsgebiet}

Als Deutsch-Südwestafrika 1884 zum ersten deutschen Schutzgebiet erklärt wurde, hatte dies zunächst keine unmittelbaren Konsequenzen für die Lebenswirklichkeit der einheimischen Bevölkerung(en). Kennzeichen dieser ersten Phase deutscher Kolonialherrschaft war weniger die Errichtung faktischer als die Dokumentation nomineller Herrschaftsverhältnisse (Krüger 1999: 32; Zimmerer 2001: 17-18). Erst mit und unter dem ersten Gouverneur Theodor Leutwein begann während der 1890er Jahre in einer zweiten Phase »die systematische Etablierung deutscher Herrschaft und der planmäßige Aufbau einer deutschen Verwaltung « (Zimmerer 2005: 26; vgl. auch Krüger 1999: 32). Parallel dazu wurden mit zumeist fragwürdigen Mitteln große Mengen Land »erworben« (Harring 2007: 439). Hierdurch wurden 1.) die Bedingungen für den massenhaften Zuzug deutscher Siedler geschaffen (Kößler/Melber 2004: 41), wurde 2.) den traditionellen politischen Autoritäten ihre ökonomische Machtgrundlage genommen und damit die Unterwerfung der einheimischen Bevölkerung unter das deutsche Gewaltmonopol vorangetrieben (Zimmer 2001: 25) und wurde schließlich 3.) die afrikanische Bevölkerung ihrer Existenzgrundlage beraubt und damit als billiges Arbeitskräftereservoir für die Siedlerökonomie erschlossen (Kößler/Melber 2004: 42-43).

Die fortwährende Demütigung, welche die Kolonialisierung für die namibische Bevölkerung bedeutete - die Ohnmacht gegenüber den betrügenden, misshandelnden, vergewaltigenden, mordenden deutschen »Herrenmenschen «, die massiven Landverluste sowie die spürbare Erosion der eigenen wirtschaftlichen und sozialen Strukturen - nährte Unzufriedenheit und provozierte schließlich Widerstand (Madley 2004: 183-184; Zimmerer 2001: 31). Als es Anfang 1904 zum sogenannten »Herero-Aufstand« kam, bemühte sich Leutwein trotz einer aufgebrachten Siedler- 
schaft zunächst noch um eine friedliche Konfliktlösung (Gewald 1996: 204), wobei er neben praktischen vor allem ökonomische Gründe gegenüber jenen »unüberlegten Stimmen « geltend machte, »welche die Hereros nunmehr vollständig vernichtet sehen wollen « - »nur politisch tot« wollte auch der Gouverneur das Volk sehen (Drechsler 1966: 173).

In Berlin entschied man sich jedoch - unter Beteiligung Wilhelms II. (Gewald 2005: 70) -, die Leitung des Feldzugs aus den Händen Leutweins zu nehmen und sie Lothar von Trotha anzuvertrauen. Als dieser Deutsch-Südwestafrika erreichte, kam er zwar ohne Kenntnis von Land und Leuten, dafür aber mit der Empfehlung besonderer Kompromiss- und Erbarmungslosigkeit bei der Niederschlagung kolonialer »Aufstände« sowie mit einer klaren Vorstellung davon, dass und wie man einen »Rassenkrieg« zu führen habe. Überzeugt, dass Afrikaner »nur der Gewalt weichen «, war es seine proklamierte Politik, diese »mit krassem Terrorismus und selbst mit Grausamkeit auszuüben« und die »aufständischen Stämme mit Strömen von Blut und Strömen von Geld« zu vernichten (zit. nach Drechsler 1966: 180). Während Leutwein einen Verhandlungsfrieden im Sinne seiner kolonialökonomischen Herrschaftsfantasie anstrebte (politische Vernichtung), setzte von Trotha auch schon im Voraus und weitab von der »Hitze des Gefechts« auf Vernichtung im Sinne seiner militärisch-genozidalen Fantasien (physische Vernichtung) - und er setzte sich durch (Zimmerer 2005: 30).

Als nach der sogenannten Schlacht am Waterberg im August 1904 die militärische Entscheidung zugunsten der Deutschen gefallen war, begann »die eigentlich genozidale Phase « (Zimmerer 2005: 31) des Krieges. Von Trotha ließ den Herero, die mit Frauen, Kindern und Rinderherden in die angrenzende Omaheke-Wüste flohen, nachsetzen und systematisch die wenigen Wasserstellen besetzen. Der »Feind « wurde, wie es später im unverhohlenen Pathos der offiziellen Militärgeschichtsschreibung heißt, "wie ein halb zu Tode gehetztes Wild [...] von Wasserstelle zu Wasserstelle gescheucht, bis er schließlich willenlos ein Opfer der Natur seines eigenen Landes wurde. Die wasserlose Omaheke sollte beenden, was deutsche Waffen begonnen hatten: Die Vernichtung des Hererovolkes" (Zimmerer 2005: 21). Und wo die Natur ihr Werk nicht schnell genug verrichtete und deutsche Patrouillen auf Nachzügler stießen, fiel »rechts und links ein Schuss im Dornbusch«, wie ein beteiligter Soldat ergänzt (Zimmerer 2005: 31).

Anfang Oktober 1904 erlässt von Trotha dann seine berüchtigte, später als Schießoder Vernichtungsbefehl berühmt gewordene Proklamation, in der er die ErschieBung aller aus der Wüste zurückkehrenden Herero anordnet: 
»Ich der große General der Deutschen Soldaten sende diesen Brief an das Volk der Herero. Die Hereros sind nicht mehr deutsche Untertanen. [...] Das Volk der Herero muss [...] das Land verlassen. Wenn das Volk dies nicht tut, so werde ich es mit dem Groot Rohr dazu zwingen. Innerhalb der deutschen Grenze wird jeder Herero mit oder ohne Gewehr, mit oder ohne Vieh erschossen, ich nehme keine Weiber und Kinder mehr auf, treibe sie zu ihrem Volk zurück, oder lasse auf sie schießen. Das sind meine Worte an das Volk der Herero.

Der große General des mächtigen Deutschen Kaisers« (zit.nach Drechsler 1966: 184).

Der Schießbefehl von Trothas dokumentiert eine Vernichtungsabsicht, die nichts mehr mit dem Brechen militärischen Widerstands zu tun hatte, sondern den Massenmord an Kombattanten und Nicht-Kombattanten, Frauen und Männern, Kindern und Alten anzeigt (Madley 2004: 187).

In der Zwischenzeit hatten auch die Nama im Süden einen effektiven Guerillakrieg begonnen (Kößler/Melber 2004: 44), dessen militärisch-praktische Erfordernisse sich zusammen mit der Angst um das deutsche Nationalprestige maßgeblich für die Aufhebung des Schießbefehls im Dezember 1904 verantwortlich zeichnete (Zimmerer 2005: 34). Die deutsche Vernichtungspolitik setzte sich allerdings in der nun eingeleiteten massenhaften Internierung von Herero und Nama in sogenannten »Konzentrationslagern« fort (Zimmerer 2005: 37). Sterberaten von teilweise über $50 \%$ und vorgedruckte Totenscheine mit der Aufschrift »Tod durch Entkräftung« verweisen auf den systematischen Vernichtungscharakter des kolonialdeutschen Lagersystems (Zimmerer 2005: 41; Zeller 2011). Von geschätzt 80.000 bis 100.000 Herero, die vor Kriegsbeginn in Südwestafrika lebten, wurden im Nachkriegszensus nurmehr 15.130 gezählt. Von den rund 20.000 Nama überlebten nur etwa die Hälfte Krieg und Gefangenschaft. Doch mehr noch als diese Zahlen ist es die dokumentierte Vernichtungsabsicht der verantwortlichen Akteure, welche aus Sicht der überwältigenden Mehrheit der FachwissenschaftlerInnen keinen Zweifel am Tatbestand des Völkermordes aufkommen lässt (Olusoga/Erichsen 2010; Gewald 1996; Trotha 2003; Kößler/Melber 2004; Krüger 1999; Wallace 2011; Zimmerer 2011 a).

Als Anfang 1908, Monate nach dem offiziellen Kriegsende vom 31. März 1907, die gefangenen Herero und Nama schließlich freigelassen wurden, begann die dritte und letzte Phase deutscher Kolonialherrschaft in Südwestafrika (Krüger 1999: 33 ). Gut 30 Jahre nachdem die ersten deutschen Verwaltungsbeamten südwestafrikanischen Boden betreten hatten, waren die Bevölkerungsgruppen im Zentrum und Süden des Landes »von freien, selbstständig wirtschaftenden Bewohnern ihres Landes 
zu besitzlosen, zu ihrem Überleben auf abhängige Arbeit angewiesenen Untertanen des Deutschen Reiches und seiner Vertreter geworden « (Zimmerer 2011 c: 77). Die deutsche Herrschaft in Südwestafrika währte jedoch nicht mehr lange. Bereits ein Jahr nach Ausbruch des Ersten Weltkrieges musste die Schutztruppe vor südafrikanischen Einheiten kapitulieren und läutete damit den Anfang vom, wenige Jahre später in Versailles besiegelten, Ende des deutschen Kolonialreiches ein. DeutschSüdwestafrika wurde vorübergehend in die Hände der südafrikanischen Mandatsmacht übergeben (Gewald 2005: 75-76). Diese machte jedoch keine Umstände, den vorkolonialen Status quo ante wiederherzustellen. Vielmehr setzte sich die ökonomisch-politische Unterdrückung der schwarzen Bevölkerung auch unter südafrikanischer Flagge fort (Gewald 2005: 77). Während die deutschstämmigen Siedler begannen, die deutsche »Ehre« (und die eigenen Privilegien) durch Geschichtsklitterung zu verteidigen (Gewald 2005: 78), setzte auf Seiten der Opfergruppen trotz prekärer Umstände schon früh ein bemerkenswerter Rekonstruktionsprozess ein, wobei dem Kolonialkrieg eine konstitutive Rolle zukam und zukommt (Krüger 1999: 183-264; Sarkin 2009: 183-185).

Im zeitgenössischen Kaiserreich war der Herero-Aufstand ein »regelrechtes Diskursereignis « mit einer »Flut von Publikationen unterschiedlicher Textsorten«, in welchen die Vernichtung der Herero und Nama interessanterweise zunächst »weder bestritten noch bagatellisiert wurde « (Brehl 2007: 102, 103, 116). Weiterhin trugen innenpolitische Kontroversen um den Kolonialkrieg, die ihren Höhepunkt in der Auflösung des Reichstages und den anschließenden Hottentottenwahlen von 1907 fanden, zu einer nicht zu unterschätzenden Präsenz Deutsch-Südwestafrikas im kollektiven Bewusstsein der Zeitgenossen bei. Es entstand ein regelrechter Kolonialmythos, der sich auch über den Verlust des Kolonialreichs hinaus in der "populären Imagination « (Conrad 2008: 117) konservierte und später von den Nationalsozialisten gezielt eingespannt wurde - wenngleich für diese »der Raumgewinn im Osten absolute Priorität hatte« (Speitkamp 2014: 172).

\subsection{Die deutsche Namibiapolitik von 1945 bis 1989}

Nach 1945 setzte innerhalb der deutschen Gesellschaft mit Bezug auf die koloniale Vergangenheit ein bis heute spürbarer »Prozess des Verschweigens und Verdrängens ein « (Kößler 2011: 80). In der Außenpolitik der jungen Bundesrepublik spielte Namibia ebenfalls so gut wie keine Rolle - Südwest war allenfalls ein »Tummelfeld reiselustiger Konservativer « (Verheugen 1986: 220-221). Man schien sich in Bonn »mit dem Status quo abgefunden zu haben, daß Namibia - die Bezeichnung hatten die UN 1968 aus der Taufe gehoben - wie eine fünfte Kolonie von Südafrika 
verwaltet wurde« (Verheugen 1986: 220). Erst als die Befreiungsbewegung SouthWest Africa People's Organisation (SWAPO) 1966 den bewaffneten Kampf aufnahm, als nacheinander die Vollversammlung der Vereinten Nationen, der UN-Sicherheitsrat und schließlich der Internationale Gerichtshof die Anwesenheit Südafrikas in Namibia für illegal erklärten und als man 1973 schließlich selbst Mitglied der Vereinten Nationen wurde, »besann sich auch die Bundesrepublik auf ihre Verantwortung gegenüber ihrer ehemaligen Kolonie« (Verheugen 1986: 220; vgl. auch Brenke 1989: 272-273).

Die Namibiapolitik der Bonner Regierungen war in den 1970er und 1980er Jahren von einem geradezu chronischen Auseinanderfallen von werteorientierter Programmatik und interessenorientierter Politik geprägt. Besonders die $»$ Verflechtung der Namibia-Frage mit dem Südafrika-Problem« (Bley 1996 a: 202) machte Bonn anfällig für außenwirtschaftlichen Opportunismus. Ganz nach dem Motto Willy Brandts, Politik und Handel nicht ohne Not zu koppeln, sprach man sich sonntags - nicht zuletzt mit Blick auf die afrikanische Mehrheit in den Vereinten Nationen gegen Rassendiskriminierung und für das Selbstbestimmungsrecht der Völker aus, nur um sich wochentags an den guten Wirtschaftsbeziehungen zu Südafrika zu erfreuen (Brenke 1989: 273). Es war jedoch nicht nur wirtschaftliches Interesse, sondern auch die »Fürsorgepflicht« für die Namibiadeutschen, die für das doppelte Spiel der Bonner Namibiapolitik verantwortlich zeichnete. So unterhielt die Bundesrepublik als einziger Staat bis Mitte der 1970er Jahre ein Konsulat in Windhuk. Offiziell erklärte man dies mit konsularischer Unterstützung für die Namibiadeutschen, faktisch bestätigte man damit das südafrikanische Apartheid- und Besatzungsregime in seinen Gebietsansprüchen über Namibia (Melber 1987: 47). Später wurde die Sorge um die Namibiadeutschen zum Herzensanliegen einer für die deutsche Außenpolitik einzigartigen »Südwester-Lobby« (Haspel 1995: 187-209; Melber 1987). Die »Südwester « unterstützten, anders als die große Mehrheit des namibischen Volkes und - weniger verwerflich, aber umso überraschender - anders als die offizielle deutsche Außenpolitik unter Genscher, die auch von Südafrika bevorzugte »intern[e] Lösung « und damit eine Marionettenregierung Pretorias, während die SWAPO ironischerweise als Terrorgruppe an den Fäden Moskaus verteufelt wurde (Verheugen 1986: 224-225).

Die Bundesrepublik beteiligte sich also einerseits als Mitglied der westlichen $»$ Kontaktgruppe« an der Erarbeitung einer friedlichen Konfliktlösung unter Einbeziehung der SWAPO. Diese Bemühungen mündeten in die Verabschiedung der UNSicherheitsratsresolution 435, welche den Weg in die namibische Unabhängigkeit bereiten sollte (Brenke 1989: 274; Verheugen 1986: 221). Andererseits aber tolerierte Bonn aus Rücksicht gegenüber dem amerikanischen Verbündeten sowohl die 
südafrikanische »Verzögerungstaktik « (Melber 1987: 45), die den namibischen Befreiungskrieg um ein sinnloses Jahrzehnt verlängerte, als auch die Nebenaußenpolitik der »Südwester «, aus deren Kreisen eine »interne Lösung « auch weiterhin gefordert und gefördert wurde (Melber 1987: 48-50; Verheugen 1986: 232-234).

\subsection{Die deutsch-namibischen Beziehungen seit der namibischen Unabhängigkeit}

Als Namibia am 21. März 1990 nach über einem Jahrhundert der Fremdherrschaft schließlich seine Unabhängigkeit feiern durfte, ging die SWAPO als erwartet klarer Sieger aus den Wahlen hervor. In Bonn bemühte man sich eilends, die eigene, vom Gros der Beobachter als »doppelbödig « (Bley 1996 a: 201) oder »doppelzüngig« (Haspel 1995: 187) eingeschätzte Rolle im Unabhängigkeitsprozess in ein gutes Licht zu rücken und zugleich die Grundpfeiler der zukünftigen Namibiapolitik abzustecken. Als wegweisend erwies sich dabei die Bundestagsdebatte vom 24. Februar 1989. Die daraus hervorgegangene Beschlussempfehlung fungierte lange und im Grunde bis heute als eine Art Verfassungsdokument deutscher Namibiapolitik. Dort bekennt man sich zu seiner »besonderen Verantwortung für Namibia«, erklärt, der neue Staat werde ein »besonderer Schwerpunkt deutscher Entwicklungszusammenarbeit« sein und verspricht schließlich auch, sich »für die berechtigten Interessen der deutschsprachigen Minderheit in Namibia einzusetzen" (Deutscher Bundestag 1989 b). Das Thema Genozid wurde in beidseitigem Einvernehmen aus den bilateralen Beziehungen beider Länder herausgehalten und - nachdem es lange integraler Bestandteil der nationalen Befreiungsgeschichte gewesen war - »unmittelbar nach der Unabhängigkeit Namibias zum 〉Spezialgebiet` der Herero-Eliten« (Gewald 2005: 86). Und diese begannen früh und lautstark, Reparationen für den im deutschen Namen verübten Völkermord an die Adresse der Bundesrepublik als Rechtsnachfolgerin des Deutschen Reiches zu richten.

Während sich Helmut Kohl bei einem Staatsbesuch 1995 noch weigerte, überhaupt mit Vertretern der Herero zu sprechen, wusste Bundespräsident Roman Herzog, als er Namibia drei Jahre später besuchte, bereits darauf hinzuweisen, dass die Verbrechen des Kaiserreichs zum damaligen Zeitpunkt nicht gegen geltendes Völkerrecht verstoßen hätten, wenn sie auch »nicht in Ordnung « waren (zit. nach Eicker 2009: 83). Bis in die jüngste Vergangenheit wurde seitdem von allen Bundesregierungen gebetsmühlenartig wiederholt, dass die Konvention zur Verhütung und Bestrafung des Völkermords vom 9. Dezember 1948 für die Bundesrepublik erst am 22. Februar 1955 in Kraft getreten sei und daher gemäß dem Grundsatz des intertemporalen Völkerrechts (Eicker 2009: 98) kein Straftatbestand vorliege, auf den 
sich etwaige Entschädigungsforderungen stützen könnten (vgl. etwa Deutscher Bundestag 2012 d). Ihrer besonderen historischen und moralischen Verantwortung für Namibia ist sich die Bundesrepublik gleichwohl bewusst und kommt dieser mit den pro Kopf höchsten Entwicklungshilfezahlungen in Afrika nach. Er empfinde, so bringt der damalige Außenminister Joschka Fischer die langjährige Haltung der Bundesregierung auf den Punkt, »tiefes Bedauern« für die damals in deutschem Namen verübten Verbrechen, nur werde er »keine Äußerung vornehmen, die entschädigungsrelevant wäre« (zit. nach Grill 2004).

Als die Herero Ende der 1990er Jahre bemerkten, dass sie mit ihren Forderungen auf formaljuristischen Granit bissen, beschlossen sie unter Federführung ihres Paramount Chief Kuaima Riruako, die Bundesregierung rechtlich fühlen zu lassen, was sie politisch nicht hören wollte. Nachdem zunächst eine gegen den deutschen Staat gerichtete Klage wegen Völkermordes beim Internationalen Gerichtshof in Den Haag aufgrund fehlender Parteifähigkeit der Herero - nur Staaten können gegen andere Staaten Klage einreichen - abgewiesen wurde (Kämmer/Föh 2004: 298), strengten die Herero 2001 vor einem US-Zivilgericht weitere Klagen gegen die Bundesrepublik Deutschland und drei deutsche Unternehmen (Deutsche Bank AG, Torex Corporation und Woermann-Linie) auf je zwei Milliarden US-Dollar Wiedergutmachung an. Die Bundesrepublik verweigerte unter Berufung auf den Grundsatz der Staatenimmunität die Zustellung der Klage, die mangels Zuständigkeit bis heute juristisch erfolglos bzw. folgenlos blieb (Eicker 2009: 329; Sarkin 2009). ${ }^{4}$

Politisch war die Klage dagegen alles andere als folgenlos, hängt sie doch wie ein Damoklesschwert über den deutsch-namibischen Beziehungen und zieht geschichtspolitische, juristische und diplomatische Probleme sowie jede Menge-auch internationale - Aufmerksamkeit auf sich (Harring 2007: 449-450; Krüger 2003: 137). Die Bundesregierung vermied daher lange vehement, das Wort Völkermord überhaupt in den Mund zu nehmen, geschweige denn sich dafür »entschädigungsrelevant « zu entschuldigen. Die bis vor Kurzem einzige Ausnahme von dieser ehernen Regel deutscher Namibiapolitik bildete die viel be- und geachtete Rede, welche die damalige Bundesministerin für Entwicklung und wirtschaftliche Zusammenarbeit Wieczorek-Zeul im August 2004 anlässlich des 100. Jahrestages der Schlacht am Waterberg in Okakarara gehalten hat und bei der sie erstmals das »amtliche Benennungsverbot« (Kößler 2011: 82) durchbrach:

4 Während für manche das Völkerrecht trotz des »zynische[n] Beigeschmack[s]« der Feststellung, dass der Völkermord an den Herero legal war und zwar nach Regeln, die zum Nachteil und ohne Beteiligung der Kolonialvölker gestaltet worden waren, nicht als Instrument der Wiedergutmachung taugt (Kämerer/Föh 2004, hier: 317; Eicker 2009), ist für andere Autoren auch der Rechtsweg nicht grundsätzlich verbaut (Anderson 2005; Jaguttis 2010; Sarkin 2009). 
»Die damaligen Gräueltaten waren das, was heute als Völkermord bezeichnet würde - für den ein General von Trotha heutzutage vor Gericht gebracht und verurteilt würde. Wir Deutschen bekennen uns zu unserer historisch-politischen, moralisch-ethischen Verantwortung und zu der Schuld, die Deutsche damals auf sich geladen haben. Ich bitte Sie im Sinne des gemeinsamen >Vaterunser« um Vergebung unserer Schuld« (Wieczorek-Zeul 2007: 47).

Obwohl auch diese Formulierung kein juristisch eindeutiges Schuldeingeständnis kommuniziert (Eicker 2009: 87; Jamfa 2008: 210-211), stellt die Rede der Ministerin den bis heute ernsthaftesten Versuch einer Bundesregierung dar, einen Versöhnungsprozess zu etablieren. ${ }^{5}$ Aus Sicht der Opfergruppen, insbesondere der Herero, wurde dieser jedoch dadurch erschwert, dass 1.) die Entschuldigung der Ministerin bis vor Kurzem nichts an der Haltung der Bundesregierung in der Reparationsfrage änderte sowie 2.), dass die später (bezeichnenderweise) in Sonderinitiative umbenannte Versöhnungsinitiative - in deren Rahmen unter Projektleitung der namibischen Nationalen Planungskommission mit ursprünglich 20 und inzwischen 31 Millionen Euro jene Gebiete (nicht Gruppen) besonders gefördert werden sollen, die vom deutschen Kolonialismus besonders betroffen waren - einseitig ins Leben gerufen wurde, ohne dass dem ein die Opfergruppen einschließender Dialog vorausgegangen wäre (Kößler 2008: 328-329).

Während man in Deutschland nach diesem »Very ambiguous $>$ I am sorry « « (Jamfa 2008) zunächst zur Tagesordnung überging, »erfuhr die Auseinandersetzung in Namibia eine deutliche Dynamisierung " (Kößler 2011: 82). Lange Zeit hatte die namibische Regierung, die seit 1990 durchgehend von der SWAPO gestellt wird, separate Reparationsforderungen mit dem (historisch falschen) Argument abgelehnt, die ganze Nation und nicht nur die Herero habe unter der deutschen Kolonialherrschaft gelitten (Harring 2007: 446). Umso überraschender nahm das namibische Parlament 2006 einstimmig eine von Riruako eingebrachte Resolution und sich damit der Reparationsforderungen der Opfergruppen an. Nach Auskunft der

5 Interessanterweise lässt die Ministerin selbst an der Interpretation ihrer Worte als Entschuldigung keinen Zweifel, was sie - jenseits des offiziellen Rededokuments - sowohl unmittelbar nach ihrer Rede mündlich als auch später schriftlich sowie im Gespräch mit uns unmissverständlich zum Ausdruck brachte: »Ich glaube Tatsachen bleiben Tatsachen und Schuld bleibt Schuld. Sicher, ich kleidete meine Bitte um Vergebung in eine christliche Formel, jenseits des juristischen Diskurses. Ich war in meiner Aussage dennoch klar und deutlich. Dass ich formulierte, ich bäte in den Worten des allen Christen gemeinsamen Vaterunser um Vergebung unserer Schuld, war das aus meiner Sicht eigentlich viel weitgehendere, als wenn ich die vier abstrakten Worte >Ich bitte um Entschuldigung` gebraucht hätte. Um aber wirklich niemanden im Zweifel zu lassen, was gemeint war, fügte ich meiner Rede spontan einen Nachsatz an: >Everything I said in my speech was an apology for the crimes committed by German Troops«" (Wieczorek-Zeul 2007: 49). 
Bundesregierung hatte sich die namibische Regierung die Parlamentsresolution Ende 2011 noch »nicht zu eigen gemacht « und die Bundesrepublik davon lediglich mit einjähriger Verzögerung in Kenntnis gesetzt, ohne sich »in der Sache zu den Entschädigungsforderungen geäußert « zu haben (Deutscher Bundestag 2011 b). Utoni Nujoma, zu diesem Zeitpunkt namibischer Außenminister, hatte dagegen zuvor beteuert: »The Namibian Government has not failed to take ownership of the demand for compensation for the 1904 to 1908 genocide« (zit. nach Sasman 2011). Fest steht, dass der damalige namibische Präsident Hifikepunye Pohamba nach einem Briefwechsel mit Angela Merkel Mitte 2013 ein hochrangig besetztes cabinet comittee unter Vorsitz des damaligen Premierministers Hage Geingob mit dem expliziten Ziel ins Leben rief, »to guide Namibia's negotiations on the issue of reparations" (Geingob 2014). Zwar scheiterte das Vorhaben am Misstrauen der Opfergruppen, die scharf gegen die Usurpierung ihrer Sache opponierten, gleichwohl ließ sich bereits dies als Hinweis auf die Wichtigkeit lesen, welche die namibische Regierung und der 2015 ins Amt gewählte Präsident Geingob dem Thema beimessen.

Auch hierzulande ist es in jüngerer Zeit zu einer im Sommer diesen Jahres kulminierenden Politisierung der Namibiapolitik gekommen. Einen Tiefpunkt bildete dabei die Rückführung von während der Kolonialzeit zu »wissenschaftlichen" Zwecken nach Deutschland gebrachter menschlicher Gebeine. Die Bundesregierung war bei der offiziellen Übergabezeremonie an eine hochrangig besetzte namibische Delegation im September 2011 »lediglich« in Form von Staatsministerin Cornelia Pieper vertreten, was auf der protokoll- und zeremoniellbedachten namibischen Seite als Kränkung empfunden wurde (Katjavivi 2014: 162). Als Pieper in ihrer Rede abermals eine offizielle Entschuldigung vermissen ließ und die Veranstaltung »aus Sicherheitsgründen« vor deren Ende verließ, war der »Eklat« (Repräsentant der Bundesregierung 2014) perfekt.

Um die diplomatischen Wogen zu glätten, reiste Anfang 2012 der Afrikabeauftragte des Auswärtigen Amtes, Walter Lindner, nach Namibia und sprach dabei als Vertreter der Bundesregierung erstmals auch mit VertreterInnen der Opfergruppen. Die Bundesregierung bestätigte später, dass man »auch zukünftig direkte Gespräche und Kontakte mit den namibischen Volksgruppen suchen wird «, da die namibische Regierung »inzwischen Direktkontakte zwischen den Vertretern namibischer Volksgruppen und der Bundesregierung ausdrücklich« begrüßt (Deutscher Bundestag 2012 c). Daneben gibt es seit einigen Jahren Versuche, als Entsprechung zur 2012 im namibischen Parlament gegründeten namibisch-deutschen Freundschaftsgruppe auch im Bundestag eine deutsch-namibische Parlamentariergruppe auf die Beine zu stellen und damit den parlamentarischen Dialog voranzutreiben. Dieses Anliegen wurde zwar aus allgemeinen Erwägungen (»zu viele Parlamentariergrup- 
pen «) blockiert, die Tatsache aber, dass die neu gewählte Bundesregierung es den Parlamentariern überlässt, »in welchem Rahmen sie diese Kontakte in Zukunft pflegen möchten « (Deutscher Bundestag 2013), war nach der Aufnahme direkter Gespräche mit den Opfergruppen ein weiteres Indiz für einen sich abzeichnenden Wandel deutscher Namibiapolitik.

War zunächst jedoch noch davon auszugehen, dass die deutsche Namibiapolitik auch unter dem neuen und alten Außenminister Frank-Walter Steinmeier allenfalls eine partielle Rekalibrierung erfahren würde, muss spätestens nach den Ereignissen der letzten Monate von einer signifikanten Neupositionierung der Namibiapolitik der Bundesregierung gesprochen werden. Während die von Außenminister Steinmeier im Juni 2014 mit seiner namibischen Amtskollegin Netumbo Nandi-Ndaitwah vereinbarte Aufnahme eines politischen Dialogprozesses (Deutscher Bundestag 2015) anfangs noch einen vergleichsweise unspektakulären Eindruck machte, lässt sich dies nach den jüngsten Entwicklungen kaum mehr behaupten. Zunächst intensivierte die Anerkennung des Genozids an den Armenieren durch Bundestag und Bundespräsident im April 2015 den ohnehin erhöhten öffentlichen und zivilgesellschaftlichen Druck, auch den Völkermord an den Herero und Nama anzuerkennen. Anfang Juli stellte dann eine nach Deutschland gereiste (und vom Bundespräsidenten nicht vorgelassene) Delegation von Herero und Nama der Bundesregierung das Ultimatum, sie werde bis zum 2. Oktober 2015 erneut rechtliche Schritte einleiten, sollte es bis dahin zu keiner offiziellen Anerkennung des Völkermords gekommen sein.

Völlig unerwartet wurde diese Forderung wenige Tage später erfüllt. Zunächst sprach Bundestagspräsident Nobert Lammert in einem Zeitungsbeitrag davon, dass es sich bei den deutschen Massakern an den Herero und Nama an den »heutigen Maßstäben des Völkerrechts gemessen « um einen »Völkermord « handle (Lammert 2015). Tags darauf sprach mit Martin Schäfer, Sprecher des Auswärtigen Amts, elf Jahre nach Wieczorek-Zeul erstmals wieder ein Vertreter der Bundesregierung und erstmals überhaupt ein Vertreter des Auswärtigen Amtes von Völkermord. Im Rahmen einer denkwürdigen Regierungspressekonferenz erklärte Schäfer die neue Stoßrichtung der regierungsamtlichen Namibiapolitik. Die »politische Leitlinie« für die zukünftige Namibiapolitik sei von nun an der vom damaligen Oppositionsführer Steinmeier unterzeichnete Antrag der Fraktionen SPD und Grüne vom 20.3.2012, in welchem von »Kriegsverbrechen und Völkermord « die Rede ist (Deutscher Bundestag 2012 a). Auf Grundlage dieser »Geisteshaltung« sei man vor gut einem Jahr in einen Dialog mit der namibischen Regierung getreten, um »ein gemeinsames Verständnis über das, was geschehen ist, zu gewinnen« (Bundesregierung 2015). Eine Entschuldigung wurde gleichwohl (noch) nicht ausgesprochen und es blieb 
zum Zeitpunkt der Fertigstellung des Beitrages offen, inwiefern die historische Bewertung des Völkermords als Völkermord auch juristische Folgen haben wird (skeptisch: Kaurasia 2015) und inwiefern neben dem namibischen Staat auch die RepräsentantInnen der vom Völkermord betroffen Gemeinschaften in die Gespräche und Entscheidungsfindung einbezogen werden (skeptisch: Johnson 2015).

\section{Die Rekonstruktion der deutschen Namibiapolitik und ihre Implikationen für eine Theorie deutscher Außenpolitik}

Gewissermaßen die heuristische Nullhypothese dieser Arbeit, ihre zu prüfende und zu verunsichernde Ausgangsvermutung, ist die unspektakuläre Annahme, dass die grundlegende Eigenschaft deutscher Außenpolitik »nicht in der konsequenten Orientierung des eigenen Handelns am Rollenskript einer Zivilmacht, sondern in der kontinuierlichen Priorität deutscher Interessen « besteht (Roos 2012: 34). Um unsere Ergebnisse vorwegzunehmen: Wir sehen diese Hypothese in der deutschen Namibiapolitik grundsätzlich bestätigt. Unser übergreifender Befund lautet, dass sich die Namibiapolitik der Bundesregierung im Kern am nationalen Interesse Deutschlands orientiert, wenngleich dieses Interesse durchaus mehrdimensional definiert und flexibel verfolgt wird. Um diesen Befund zu plausibilisieren, werden wir auf den folgenden Seiten zu zeigen versuchen, dass die deutsche Namibiapolitik 1.) im Wesentlichen eine Politik der Bundesregierung, gleichwohl aber nicht unabhängig von (partei-)politischen Kräfteverhältnissen ist; dass sie 2.) an der Wahrung und Förderung deutscher Interessen orientiert ist; dass diese Interessen aber 3.) auf differenzierte Weise definiert und verfolgt werden, was es wiederum erforderlich macht, die kooperativen, kompromissbereiten und vergangenheitsbezogenen Aspekte deutscher Namibiapolitik in unserer These vom Primat nationaler Interessen zu integrieren.

\subsection{Namibiapolitik zwischen regierungsamtlicher Prägung und (partei-)politischer Beeinflussung}

Dass wir von einer relativen Deckungsgleichheit der Namibiapolitik des deutschen Staates und der Namibiapolitik der Bundesregierung ausgehen, lässt sich dadurch begründen, dass wir Rhetorik und Richtung der deutschen Außenpolitik gegenüber Namibia als maßgeblich von den außenpolitischen »Apparaten«-insbesondere dem Auswärtigen Amt - vorgegeben sehen. Diese agieren zwar nicht im parteipolitisch und zivilgesellschaftlich luftleeren Raum, haben aber zumindest eine gewisse Unabhängigkeit von Legislaturperioden und politischem Tagesgeschäft. Gleichwohl müssen sie sich - und das hat die Neupositionierung der Bundesregierung im Juli 
2015 vor dem Hintergrund massiven zivilgesellschaftlichen und parlamentarischen Drucks eindrucksvoll gezeigt - gegenüber den jeweiligen politischen Kräfteverhältnissen durchaus responsiv zeigen. Es ist daher sicherlich kein Zufall, dass die jüngsten Veränderungen in der deutschen Namibiapolitik 1.) unter einem sozialdemokratischen Außenminister erfolgt sind, der sich als Oppositionsführer selbst noch für eine Anerkennung des Völkermords eingesetzt hatte, und dass sie 2.) zu einem Zeitpunkt erfolgt sind, zu dem der öffentliche Ruf nach Anerkennung des Völkermords - man denke nur an den vielfach und prominent unterzeichneten Appell Völkermord ist Völkermord oder die mediale Intervention Norbert Lammerts - kaum mehr zu überhören war.

Ungeachtet dessen lässt sich die langjährige Haltung der Bundesregierung und bis zu einem gewissen Grad auch die vor Kurzem erfolgte Änderung dieser Haltung nur bedingt mit parteipolitischen Unterschieden in der Zusammensetzung der Regierung erklären. Diese verschwinden zwar nicht, sobald sie in Regierungshandeln übersetzt werden, werden aber doch relativiert. Namibiapolitik war und ist in der Überzeugungsstruktur der Bundesregierung und des Auswärtigen Amtes eine Frage des langfristigen nationalen Interesses und daher Sache der Berufsaußenpolitiker, die zwar nicht über gesellschaftlichen Einzelinteressen stehen, diesen aber auch nicht einfach unterworfen sind.

\subsection{Deutsche Namibiapolitik als Interessenpolitik}

Mit Blick auf die Frage, was nun das nationale Interesse in der deutschen Namibiapolitik definiert, muss unsere Antwort zunächst scheinbar tautologisch ausfallen: Es ist das, was die maßgeblichen (außenpolitischen) Akteure darunter verstehen. Diese Antwort ist aber genau deswegen nicht trivial, weil sie bereits auf das Selbstverständnis der beteiligten Akteure verweist. Eine zumindest in der Namibiapolitik dominante Strömung innerhalb der Bundesregierung versteht die Bundesregierung als einen Akteur, der ein immer zugleich vorausgesetztes und fortlaufend mit- und neudefiniertes Interesse des deutschen Nationalstaates möglichst gewinnbringend nach außen hin vertritt. Und so können auch wir die Bundesregierung verstehen. Als eine Art »Bank des deutschen Nationalinteresses«, deren selbstverstandene Aufgabe es ist, das Staatswohl der Bundesrepublik in der Welt zu verwalten und zu mehren.

Wichtiges Element einer solchen Politik ist der kluge Umgang mit der Völkermord- und Reparationsfrage. Dies bedeutet aus Sicht der Bundesregierung einerseits die Zahlung von Reparationen zu verhindern, andererseits die Schaffung eines Präzedenzfalls zu vermeiden, welcher deutsche Firmen oder westliche 
Partnerregierungen in die Verlegenheit bringen könnte, ebenfalls für die eigene koloniale Vergangenheit juristisch und finanziell gerade stehen zu müssen:

»Gut, ich sag auch, die Haltung der Bundesregierung, auch die rechtliche Haltung, ist unverändert. Denn Sie müssen sich vorstellen, wenn man sich entschuldigen würde, hieße das, dass man auch zahlen muss und das will man aus vielerlei Gründen vermeiden: aus Präjudizgründen, ich sag das auch offen, auch im Hinblick auf frühere Kolonialmächte, und es würde eine Unsumme von Klagen auch auf deutsche Firmen zukommen. Und das was wir tun, ich sag das auch, wir vertreten unsere Interessen« (Repräsentant der Bundesregierung 2014).

Eine wirklich entschädigungsrelevante Aussage wurde und wird also - sofern und solange es politisch irgendwie zu rechtfertigen ist - aus Kosten- wie aus »Präjudizgründen « vermieden, da beides dem wohl so verstandenen Eigeninteresse des deutschen Nationalstaates schadet. In diesem Verständnis markiert auch die jüngste Verwendung der Völkermordbegrifflichkeit keine so grundsätzliche Änderung der regierungsamtlichen Haltung, wie man dies auf den ersten Blick vermuten könnte. Schließlich will man die Verwendung des Völkermordbegriffs zunächst einmal historisch und nicht rechtlich verstanden wissen und die Frage einer möglichen Entschuldigung bislang ebenfalls - und auch auf explizite Nachfrage hin - ungeklärt lassen (Kauraisa 2015). Und selbst wenn eine solche am Ende des politischen Dialogprozesses mit Namibia stehen sollte, scheint es uns nicht nur fraglich, ob sich daraus tatsächlich ernsthafte juristische Konsequenzen ergeben würden, sondern auch, ob darauf direkte Verhandlungen mit den Opfergruppen folgen würden. Bei der nun erfolgten Anerkennung des Völkermordes in einem historischen Sinn handelt es sich vor diesem Hintergrund also eher um einen - wenn auch begrüßenswerten - Strategie-, als um einen fundamentalen Kurswechsel.

Das Primat nationalstaatlicher Interessen äußert sich in der deutschen Namibiapolitik vor allem als an politischen und wirtschaftlichen Kalkülen orientierte Vorteilsmehrung. Das Bild des kompetitiv-kaltschnäuzigen »Brokers des deutschen Staatswohls« verdankt seine Plausibilität der Rekonstruktion einer Grundhaltung der Bundesregierung. Gemäß dieser ist die koloniale Vergangenheit eine Art»faules Papier« im Keller, das man zwar nicht mehr loswird, das man aber mehr oder weniger verlustreich verwalten, von dem man in Zukunft vielleicht sogar wieder eine Rendite erwarten kann. Moralisch-ethische Prinzipien nehmen dabei gewissermaßen den Stellenwert von Compliance-Regeln ein: Man hat sich zwar grundsätzlich an sie zu halten, sie spielen im operativen Tagesgeschäft aber nur dann wirklich eine Rolle, wenn (öffentlicher) Druck auf ihre Einhaltung drängt bzw. wenn ihre Nicht- 
Einhaltung mit Blick auf die eigenen Ziele zu einem Problem wird. Dies zeigte sich in Reaktionen der Bundesregierung auf die Wiedergutmachungsforderungen der deutschen Zivilgesellschaft, über die man offenkundig alles andere als glücklich war (und ist):

»Aber das Thema der Wiedergutmachung war damit durch. Man muss schon feststellen, dass es ständig von Kräften innerhalb der Bundesrepublik Deutschland neu aufgemischt wird. Das ist nicht hilfreich für den weiteren Prozess in Namibia selber, und es ist mit Sicherheit nicht im Interesse der Bundesrepublik Deutschland« (Staatsminister Hoyer, zit. nach Deutscher Bundestag 2011 a).

Es macht(e) dabei mitunter den Eindruck, als wären der Bundesregierung jene Teile der deutschen Gesellschaft, die das Thema Völkermord und Reparationen immer wieder zu einem solchen machen, geradezu ein Dorn im Auge. Man scheint in ihnen so etwas wie Störenfriede zu sehen, die den von der offiziellen Politik angestrebten »Schlussstrich « stets aufs Neue verhindern und damit alles andere als »im deutschen Interesse« handeln. Diese Diskreditierung abweichender Positionen, nämlich solcher, »die die Vergangenheit in den Mittelpunkt stellen« (Staatsminister Günter Gloser, zit. nach Deutscher Bundestag 2008), verweist auf das Selbstverständnis der Bundesregierung, nicht nur in privilegierter Weise das nationale Interesse Deutschlands zu vertreten, sondern dieses auch in privilegierter Weise zu kennen und somit legitimerweise verfolgen zu können. Zugleich bestätigt sich darin, dass die Unabhängigkeit, die außenpolitische Akteure unterhalb des Radars der medialen Aufmerksamkeitsschwelle genießen, im Zuge der öffentlichen Politisierung eines Themas signifikant zusammenschrumpfen kann. Als der politische Druck zu groß wurde und die jahrzehntelange Position der Bundesregierung selbst im Regierungslager rasant an Unterstützung verlor, wurde ein (womöglich zuvor bereits angedachter) Strategiewechsel eingeleitet, um die grundsätzlichen außenpolitischen Ziele auch unter veränderten gesellschaftspolitischen Bedingungen erreichen zu können.

Bis in die jüngere Vergangenheit war es jedoch ein vergleichsweise probates, weil nur punktuell Widerstand hervorrufendes Mittel, den Völkermordbegriff in offiziellen Sprechakten konsequent zu vermeiden. Dieses »Herumwinden« um den Völkermordbegriff zog sich mit Ausnahme von Heidemarie Wieczorek-Zeul ${ }^{6}$ bis zur

6 Es kann davon ausgegangen werden, dass Wieczorek-Zeul ihre Rede im Jahr 2004, die sie nach eigenem Bekunden »im Namen der Bundesregierung« (Wieczorek-Zeul 2007: 42) gehalten hat, zuvor nicht mit dem Auswärtigen Amt und en détail möglicherweise auch nicht mit dem Kanzleramt abgesprochen hat. Zumindest schloss der damalige Botschafter Wolfgang Massing eine Entschuldigung unmittelbar vor Wieczorek-Zeuls Rede explizit aus (Wieczorek-Zeul 2007: 48) und war wohl 
Regierungspressekonferenz vom 10. Juli 2015 wie ein roter Faden durch die deutsche Namibiapolitik. Noch 2011 vermied Cornelia Pieper (2011) in ihrer Rede anlässlich der Feierstunde zur Übergabe von Schädeln namibischen Ursprungs den Begriff Völkermord und sprach stattdessen, wie bis dahin von der Bundesregierung gewohnt, von der »blutigen Niederschlagung der Aufstände«. Diese Praxis hat begrüßenswerter Weise im Zuge der sich immer stärker dynamisierenden, medialen und zivilgesellschaftlichen Politisierung des Themas und der damit einhergehenden massiven Glaubwürdigkeitskrise deutscher Namibiapolitik ein (hoffentlich dauerhaftes) Ende gefunden.

Wäre die deutsche Namibiapolitik jedoch immer schon Ausdruck einer genuinen »Werteorientierung der deutschen Außenpolitik« (Hans Büttner (SPD), zit. nach Deutscher Bundestag 2004) gewesen, ließe sich die jahrzehntelange, mitunter an Geschichtsklitterung grenzende Euphemisierung der Vergangenheit (Böhlke-Itzen 2004; Grill 2004) kaum erklären. Aus Perspektive einer im Kern an den Grundsätzen einer Zivilmacht orientierten Namibiapolitik hätte man vielmehr erwarten dürfen, dass die Frage der Einordnung und Wiedergutmachung des historischen Unrechts gegenüber den Herero und Nama gar keine ist [und nie hätte eine sein dürfen, Anm. UR/TS] - gerade weil sie eine politisch-moralische Dimension hat « (Engert 2009: 295). Eine zivilmächtige Namibiapolitik wäre schließlich kaum bereit, um der Minimierung der juristischen Angriffsfläche willen den eigenen ethischen Spielraum selbst dann bis an die Grenze des politisch Möglichen auszureizen, wenn damit im Ergebnis die Leugnung eines historischen Verbrechens und die Verweigerung von Anerkennung und glaubwürdiger Entschuldigung verbunden wären. Mit anderen Worten: Wo, wenn nicht in der Namibiapolitik, hätte eine zivilmächtige deutsche Außenpolitik von Beginn an von der »Bereitschaft « geprägt sein müssen, »Normen zu entwickeln, die allgemeinen Interessen, nicht ihren Sonderinteressen verpflichtet sind « (Maull 1992: 273). Wäre es einer Zivilmacht nicht angemessen sich bei der Verwirklichung einer von den Prinzipien ziviler Macht geprägten und daher als gerecht und fair empfundenen Weltordnung »überproportional selbst « einzubringen (Maull 1992: 277)?

\subsection{Die Mehrdimensionalität und Flexibilität der Interessenverfolgung}

Die bisher von uns formulierten Thesen nahmen in erster Linie die materielle Dimension der Interessengeleitetheit deutscher Namibiapolitik in den Blick. Wir glau-

unmittelbar danach dementsprechend »baff«. Dass Wieczorek-Zeul auf dem Rückweg nach Windhuk um ihren Ministerposten fürchtete (Wieczorek-Zeul 2007: 49), ist ein starkes Indiz, wie groß der außenpolitische Konformitätsdruck selbst für Regierungsmitglieder gewesen ist und sein kann. 
ben, dass eine solche, auf die Dominanz nationaler Interessen im engeren Sinne abstellende Erklärung der Namibiapolitik der Bundesregierung eindeutig aus dem Material hervorgeht und trotz ihrer offenkundigen Schlichtheit in hohem Maße plausibel ist. Wir glauben jedoch zugleich, dass es gute Gründe gibt, ein differenzierteres Bild zu zeichnen, welches die Hypothese juristischer und finanzieller Eigeninteressen zugleich einschließt und über sie hinausgeht. Deutsche Namibiapolitik, so die diesbezügliche These, orientiert sich zwar im Zweifel am Vorrang nationaler Interessen, weist aber zugleich auch Elemente mehrdimensionaler Interessendefinition und flexibler Interessenverfolgung auf. Konkret bedeutet dies, dass die deutsche Namibiapolitik 1.) durchaus eine kooperative Dimension hat, dass jedoch nur mit jenen kooperiert wird, mit denen man das muss; dass sie weiterhin 2.) eine pragmatische Politik ist, welche die Faktizität normativer Erwartungen zwar ernstnimmt, aber jeweils nur so ernst wie sie es gerade muss; dass sie schließlich 3.) eine zukunftsgerichtete Politik ist, welche die Vergangenheit zwar zur Kenntnis nimmt, aber nur insoweit sie das eben muss. Deutsche Namibiapolitik ist also durchaus partnerorientiert, entgegenkommend und vergangenheitsbezogen. Sie ist jedoch nur partnerorientiert, um sich unerwünschte Partner vom Leibe zu halten, nur entgegenkommend, um es an anderer Stelle nicht sein zu müssen und nur vergangenheitsbezogen, weil sie die Zukunft im Blick hat.

\subsubsection{Selektive Kooperativität oder: Der deutsch-namibische »Burgfrieden«}

Wenn man nach der (selektiven) Kooperativität deutscher Namibiapolitik fragt, stößt man zunächst auf die vergleichsweise hohen Entwicklungshilfezahlungen; besser gesagt: man wird mit an Sicherheit grenzender Wahrscheinlichkeit von VertreterInnen der Bundesregierung darauf gestoßen. Doch einmal abgesehen davon, dass es sich bei den vielzitierten 800 Millionen Euro um einen über 25 Jahre gestreckten Bruttobetrag handelt, stellt sich die sehr konkrete Frage, wer eigentlich der Adressat dieser Zahlungen ist - und wer nicht. Hier stoßen wir auf die Problematik, dass in Namibia nicht Staat und Nation, sondern eher schon - und ungeachtet der zunehmend sichtbaren Grenzen der traditionellen diskursiven Gleichsetzung von Nation und der Befreiungsbewegung SWAPO (Du Pisani 2010) - Staat und Partei in eins fallen (Woeller 2005: 193-224). Anders formuliert: Da Entwicklungsgelder zunächst einmal Verfügungsmittel für die mit komfortabler Zwei-Drittel- bis Drei-Viertel-Mehrheit regierende SWAPO sind (»Staat = Partei $\ll)$, kommen sie nicht der namibischen Nation, sondern in erster Linie der ethnischen Wähler- und Rekrutierungsbasis der SWAPO zugute (»Staat $\neq$ Nation $«)$ (Eicker 2009: 90; Jamfa 2008: 113). 
Diese befindet sich jedoch vor allem bei den im Norden des Landes beheimaten Ovambo, welche zwar gut die Hälfte der namibischen Bevölkerung stellen (CIA 2013), vom deutschen Kolonialismus aber nur am Rande betroffen waren (Gewald 2005: 89). Es ist daher gerade nicht so, dass jene Gruppen, für die Deutschland sich historisch und moralisch in besonderer Weise verantwortlich fühlen sollte, auch in besonderer Weise von der deutschen Entwicklungshilfe profitieren würden. Vielmehr schien und scheint weiterhin eine »latente Komplizenschaft « (Kößler/Melber 2004: 61), ja eine Art »Burgfrieden« zwischen deutscher und namibischer Regierung mit Blick auf Zurückweisung von separaten Entschädigungsforderungen zu existieren. Indem die Bundesregierung mit der namibischen Regierung - immer auch, das gilt es zu betonen, auf deren Wunsch hin - kooperiert, muss sie dies nicht mit den Opfergruppen tun oder riskieren, dass sich eine enttäuschte namibische Regierung deren Forderungen annimmt. Diese Form der selektiven oder ausschließenden Kooperation im Rahmen eines Burgfriedens zwischen den Regierungen bedeutet konkret, dass die SWAPO-Regierung Ressourcen erhält, um ihre innenpolitische Machtposition zu festigen, während die Bundesregierung wiederum keine zwischenstaatlichen Reparationsforderungen fürchten muss und zur Abwehr möglicher Kritik auf die hohen Entwicklungszahlungen an ein vermeintlich monolithisches Namibia verweisen kann.

Weil und solange die politische Landschaft Namibias von ethnischen cleavages durchzogen ist, kommt eine Stärkung der Opfergruppen (insbesondere der Herero) aus Sicht der SWAPO einer Stärkung der Opposition gleich (Schaller 2011: 273-274). Dies gilt umso mehr, als die Reparationsfrage eng mit der Landfrage verknüpft ist ${ }^{7}$ und die SWAPO-Regierung einen Machtzuwachs der Opfergruppen fürchten muss, sollten diese durch Reparationen in die Lage versetzt werden, Teile ihres ursprünglichen Landes zurückzukaufen (Harring 2007: 448; Woeller 2005: 196). Ebenso wenig wie der namibischen Regierung an einem Erstarken tribal-»parastaatlicher« Strukturen gelegen ist, ist die Bundesregierung an einer »radikalen« Landreform zuungunsten der deutsch(stämmig)en Landbesitzer interessiert. Es ist ja gerade dieses aufgrund seiner Offensichtlichkeit gerne übersehene Interesse, das

7 »Deutschland schuldet uns Reparationen. Sonst bleibt uns nur noch der simbabwische Weg offen. [...] Als die Deutschen das Land und Vieh nahmen, nahmen sie es den Herero weg. Deshalb ist die namibische Landfrage eine Herero-Frage« (Riruako, zit. nach Kößler/Melber 2004: 60-61). 
eine wichtige $»$ Facette« (Repräsentant der Bundesregierung 2014) ${ }^{8}$ der deutschen Namibiapolitik - und ein Distinktionsmerkmal zur Außenpolitik gegenüber den Nachfolgestaaten anderer ehemaliger Schutzgebiete, in denen es keine nennenswerten deutschen Minderheiten gibt und für die man sich offenbar nicht in derselben Weise verantwortlich fühlt - ausmacht: Die Bundesregierung vertritt und schützt nach wie vor - und hier nimmt man inzwischen auch kein Blatt mehr vor den Mund (vgl. etwa noch: Ganns 2000) - in besonderer Weise die Interessen der gut 20.000 DeutschnamibierInnen. Wir wüssten also nicht, wieso man in der Entwicklungshilfe nicht auch eine Art »Schutzgeld « für die in Namibia lebenden Deutsch(stämmig)en sehen sollte. Dies entbehrt freilich nicht eines gewissen Zynismus, verteidigt die Bundesrepublik damit doch bis heute eine Landverteilung, die das unmittelbare Resultat der deutschen Kolonialherrschaft ist (vgl. Eicker 2009: 92-93). Es lässt sich der Eindruck nicht vermeiden, dass die Verantwortung für die auch im Weltmaßstab extreme sozio-ökonomische Ungleichheit Namibias (CIA 2013) weniger schwer zu wiegen scheint als die Verantwortung für die »lieben Landsleute«(Helmut Kohl zit. nach Schmidt-Lauber 2000: 243). Die »Sorge« um die Deutschen, aber auch - in Form einer regen Außenkulturpolitik - um »das Deutsche « verweist auf ein außenpolitisches Überzeugungssystem, in dem die »besondere Verantwortung « eben immer auch als »besonderer Einfluss « zu verstehen ist, den es als Ziel und Mittel zu erhalten gilt. ${ }^{9}$ Dass die deutsche Entwicklungshilfe dabei als eine Art »Schmiermittel « des Burgfriedens verwendet wird - »Der großen Bedeutung der deutschen Entwicklungshilfe [...] sind sich beide Regierungen bewusst« (Egon Jüttner (CDU) zit. nach Deutscher Bundestag 2012b) -, bestätigt wiederum die im Forschungsstand dominante These, dass Entwicklungspolitik als »ein Mehrzweckinstrument«

8 »Sicherlich, die Unterstützung der Deutschsprachigen ist eine Facette, aber unsere Arbeit hier, unser Auftrag hier erschließt sich eben aus unserer historischen Verantwortung, deswegen ist das auch so intensiv und ich hab auch noch nie, ich bin ja jetzt auch schon lange im Auswärtigen Amt, ein Land gesehen auf dieser schönen Welt, in dem der deutsche Einfluss so stark ist, gucken Sie mal hier aus dem Fenster raus [zählt »deutsche« Gebäude der Windhuker Innenstadt auf]« (Repräsentant Bundesregierung 2014).

9 Es zeigt sich hier erneut der bereits in früheren Arbeiten rekonstruierte Zusammenhang zwischen den Konzepten »Macht « bzw. »Einfluss « auf der einen sowie »Verantwortung « auf der anderen Seite (vgl. Baumann 2006; Roos 2010). Verantwortung bedeutet weniger Verantwortung für vergangenes Unrecht in Form seiner Wiedergutmachung im Hier und Jetzt, sondern zielt auf den Schutz der dort lebenden Deutschen, der deutschen Kultur und natürlich deutscher Interessen. Verantwortung heißt dann, den besonderen Einfluss auf den namibischen Staat, die besondere Machtposition der Bundesrepublik und letztlich auch der Deutschnamibier in Namibia zu bewahren, wobei Letzteren wohl eine Art »Brückenfunktion« (Hans Klein (CSU) zit. nach Deutscher Bundestag 1989 a) bei der deutschen Interessentransmission zugedacht ist (vgl. Schmidt-Lauber 2000). 
beschrieben werden sollte, »dessen Potential je nach Perzeption von Interessenlagen eingesetzt wird« (Messner 2011: 416; ähnlich: Nuscheler 2007: 676).

Es wäre dies nun eine klassische Win-win-Situation auf Kosten Dritter, würden diese Dritten (und deren UnterstützerInnen) nicht unentwegt auf nationaler wie internationaler Ebene Druck in eigener Sache aufbauen. Seit einigen Jahren fangen diese Bemühungen auch an Früchte zu tragen und der deutsch-namibische Burgfrieden beginnt zu bröckeln. So lässt man auf Seiten der SWAPO-Regierung mittlerweile verlauten, »that national reconciliation can be hindered significantly if the issues [of reparations] are not addressed in the comprehensive manner « (zit. nach Sasman 2011). Die Bundesregierung legte derweil - notgedrungen - ihre Berührungsängste gegenüber der namibischen Zivilgesellschaft ab und sieht im direkten Dialog mit den Opfergruppen inzwischen eine »zivilgesellschaftliche Erweiterung« (Deutscher Bundestag 2012 c) ihrer bilateralen Beziehungen zur namibischen Regierung, die - so gilt es zu ergänzen - durch den intensivierten Parlamentarierdialog auch eine "parlamentarische Erweiterung « erfahren haben. Weniger wohl als Resultat aufrichtigen regierungsamtlichen Gesinnungswandels als unter dem Druck der Ereignisse, scheint gegenwärtig die bisherige dyadische Burgfriedensbeziehung in bestimmten Bereichen um eine die Opfergruppen einschließende Dreiecksbeziehung ergänzt zu werden.

Ergänzt bedeutet allerdings nicht ersetzt. Vielmehr scheint die grundsätzliche Präferenz der Bundesregierung für zwischenstaatliche Kommunikationswege auch nach jener bahnbrechenden Regierungspressekonferenz fortzubestehen, in welcher der Völkermord an den Herero und Nama nach jahrzehntelanger Verweigerung gleichsam en passant anerkannt wurde. Direkte (Wiedergutmachungs-)Gespräche mit den Opfergruppen werden weiterhin abgelehnt und der politische (Versöhnungs-)Dialog findet überwiegend auf Regierungsebene statt. Es lässt sich allerdings die - zugegebenermaßen etwas spekulative, angesichts des wachsenden Einflusses der BRICS-Staaten in Namibia, der schwindenden Abhängigkeit von deutscher Entwicklungshilfe, des mangelnden Realitätssinns vieler Deutschnamibier sowie der Armut und Ungleichheit im Land, auch nicht unbegründete - Überlegung anstellen, dass die Neupositionierung der Bundesregierung in der Völkermordfrage nicht nur innenpolitischem Druck geschuldet war, sondern auch der veränderten Positionierung der namibischen Regierung. Dass sich das namibische Parlament den Forderungen der Opfergruppen im Jahr 2006 angenommen hatte, konnte von der namibischen Regierung zwar lange Zeit »auf niedrigem Niveau« (Wietersheim 2014) gehalten werden. In jüngerer Zeit schien die SWAPO-Regierung den parlamentarischen Auftrag jedoch - nicht zuletzt vor dem Hintergrund wachsenden innenpolitischen Drucks (und gestiegener außenpolitischer Handlungsspielräume) - 
zunehmend ernster zu nehmen (Geingob 2014). Es ist nicht unplausibel zu vermuten, dass der damalige namibische Präsident Pohamba die Bundesregierung bereits 2013 in einem Briefwechsel mit der Bundeskanzlerin über eine kurz- bis mittelfristig zu erwartende Veränderung der offiziellen namibischen Haltung in der Völkermordund Reparationsfrage $»$ informierte $\ll$.

Die Aufnahme eines zwischenstaatlichen politischen Dialogs und die jüngste Anerkennung des Völkermordes wären vor diesem Hintergrund auch als Reaktion auf das partielle Brüchigwerden des deutsch-namibischen Burgfriedens und zugleich als Versuch zu verstehen, die Gespräche soweit wie möglich auf zwischenstaatlicher Ebene zu halten und in einem öffentlich beruhigten Umfeld ablaufen zu lassen. Es scheint uns wenig plausibel, die in der Tat unerwartete Positionsänderung der Bundesregierung als plötzliche Selbsterkenntnis der auf dem zivilmächtigen Auge nur ausnahmsweise jahrzehntelang blinden außenpolitischen Apparate zu deuten. Nicht nur, dass diese im vorangegangenen Vierteljahrhundert konsequenter Verweigerung oft genug auf ihren vergangenheitspolitisch blinden Fleck aufmerksam gemacht wurde. Es ist im Rahmen einer pragmatischen Verfolgung nationaler Interessen auch durchaus plausibel, die eigene Strategie vor dem Hintergrund einer innen- wie außenpolitisch zunehmend ungünstigen Situation zu ändern und gewissermaßen das Beste aus dieser zu machen.

Ein solcher Strategiewechsel hat zum gegenwärtigen Zeitpunkt sowohl das Potenzial zu einer unkontrollierten Dynamisierung als auch zu einer kontrollierten Kanalisierung der Reparationsfrage. Idealtypisch geht die Kanalisierungs- oder Kontrollthese davon aus, dass die Anerkennung des Völkermords und unverbindliche Dialogprozesse mit den Opfergruppen »Druck aus dem Kessel« ignorierter Erwartungen und angestauter Bigotterien nimmt. Einerseits wird die Bundesregierung von der, spätestens nach der Anerkennung des Armeniergenozids durch Bundestag und Bundespräsident peinlichen, Doppelmoral in der Namibiapolitik entlastet. Andererseits können Reparationsforderungen gleichsam »verstaatlicht « und damit sowohl ernstgenommen, als auch in kontrollierte, nicht-tribale Bahnen gelenkt werden, während zugleich informelle Gespräche jenseits juristischer und diplomatisch-protokollarischer Fährnisse ermöglicht werden. So lehnt die Bundesregierung einen transgesellschaftlichen Dialog zur Reparationsfrage nach wie vor und erwartbarerweise ab, setzt aber wohl gewisse Hoffnungen auf deren Entdramatisierung im Rahmen von unverbindlichen Dialogprozessen. ${ }^{10}$

10 »Die Bundesregierung unterstützt selbstverständlich Gespräche zwischen Parlamentariern. Wir wollen aber den Eindruck vermeiden, dass durch einen institutionalisierten Dialog mit dem namibischen Parlament eine Anerkennung etwaiger Entschädigungsforderungen verbunden ist «(Staatsminister Günter Gloser zit. nach Deutscher Bundestag 2008). 
Dass ihr angesichts des erheblichen inneren wie äußeren Drucks auch gar nichts anderes übrig bleibt, als neue Dialog- und Sprachformen zu finden, verweist auf ein mögliches Kontrollproblem, ${ }^{11}$ mit dem sich die deutsche (aber auch die namibische) Regierung konfrontiert sieht, und damit auf eine zweite mögliche Konsequenz eines bröckelnden Burgfriedens: eine bilateral nicht mehr zu kontrollierende Eigendynamisierung der gegenwärtig noch im Entstehen begriffenen inner- und transgesellschaftlichen Dialog- und politischen Organisationsfor(m)en. Die Entstehung neuer Allianzen über politische Lager und geographische Grenzen hinweg und die multizentrische Politisierung des Themas in deutschen wie namibischen Medien, im deutschen wie im namibischen Parlament, in Aktionsbündnissen und Petitionsgruppen im Internet und auf der Straße haben zweifellos zur Neupositionierung der Bundesregierung beigetragen. Falls diese Eigendynamik von den Regierungen nicht wieder eingefangen werden kann oder von selbst abebbt, birgt sie das Potenzial 1.) zu einer weiteren Erhöhung der Zahl manifester Konflikte, gerade weil es an Sprechprotokollen und Interessenkonvergenz fehlt - man denke nur an die diplomatischen Turbulenzen, die regelmäßig durch den Besuch von Opfergruppen ausgelöst werden; 2.) zu einer ungewollten partiellen Parlamentarisierung des außenpolitischen Primats der Exekutive und damit einer parteipolitischen Öffnung der Namibiapolitik und schließlich 3.) zur Intensivierung einer tendenziell unerwünschten, breiten gesellschaftlichen Debatte in Deutschland über die eigene koloniale Vergangenheit.

\subsubsection{Die pragmatische (Un-)Nachgiebigkeit der deutschen Namibiapolitik}

Bis zur Anerkennung des Völkermords, im unmittelbaren Anschluss an das symbolträchtige Zentenarium des faktischen Endes deutscher Kolonialherrschaft auf namibischen Boden, war die Namibiapolitik der Bundesregierung im Kern eine der pragmatischen Unnachgiebigkeit. Unnachgiebig mit Blick auf die Fragen von Völkermord und Reparationen, pragmatisch mit Blick auf eine entgegenkommende Grundhaltung in Fragen, die angesichts der in ihnen artikulierten normativen Er-

11 Die von Staatsminister Werner Hoyer im Nachgang des »Schädeleklats« getätigten Äußerungen, wonach die namibische Delegation von Organisationen der deutschen Zivilgesellschaft »geradezu aufgestachelt« (Deutscher Bundestag 2011 a) worden sei, offenbaren neben einem äußerst merkwürdigen Afrikaner- bzw. Namibierbild auch eine gewisse Hilflosigkeit mit Blick auf die Kontrolle der Opfergruppen. Weil nicht sein kann, was nicht sein darf, nämlich dass es afrikanische Akteure gibt, die aktiv um Anerkennung des an ihren Vorfahren geschehenen Unrechts kämpfen und sich dabei eben nicht immer an das kommunikative Korsett diplomatischer Regularien halten, flüchtet man sich in Anschuldigungen an die deutsche Zivilgesellschaft - als ob die namibische Delegation erst dazu hätte aufgestachelt werden müssen, sich über das abermalige Ausbleiben einer von ihnen seit Jahrzehnten geforderten Entschuldigung zu empören. 
wartungen nicht so einfach ignoriert werden können, die aber nicht unmittelbar etwas mit (den Begriffen) Völkermord und Reparationen zu tun hatten. Man zeigte sich auch vor diesem Sommer durchaus gesprächsbereit, symbolisch wie materiell, solange nur das »V-Wort« nicht genannt und das »R-Wort« nicht gefordert wurde. Man zahlt zwar überdurchschnittlich viel Entwicklungshilfe, weist aber »eine Interpretation dieser Zahlungen als Reparationen oder gar als Schuldeingeständnis zurück « (zit. nach Engert 2009: 299). Man sagt zwar, die Sonderinitiative komme »dem was gewollt wird, am nächsten" (Repräsentant der Bundesregierung 2014), aber eine Reparationsleistung ist sie nicht.

Diese Politik ist nun zum Teil einer Politik der pragmatischen Nachgiebigkeit gewichen, da man es unter dem Druck innen- wie außenpolitischer Ereignisse inzwischen für eine mit Blick auf die eigenen Ziele pragmatischere Politik erachtet, nicht mehr nur mit »kleinen Gesten [...] eine positive Stimmung zu schaffen« (Repräsentant der Bundesregierung 2014). Ein Jahrzehnt nach Heidemarie WieczorekZeuls großer Geste will man den Begriff Völkermord nun auch als historische Beschreibungskategorie in der offiziellen Namibiapolitik verwenden. ${ }^{12}$ Im Sinne eines juristischen und juristisch relevanten Beschreibungsbegriffs scheint man dies jedoch nach wie vor nicht verstanden wissen zu wollen (Kauraisa 2015). Mit Blick auf eine mögliche Entschuldigung bittet man noch »um Geduld«, da man den Ausgang der gegenwärtig geführten Gespräche mit der namibischen Regierung nicht vorwegnehmen will (Bundesregierung 2015). Ob am Ende dieser Gespräche eine »entschädigungsrelevante« Entschuldigung stehen wird, die den Opfergruppen in einem inklusiven Verhandlungsprozess die Möglichkeit gibt, ihre Interessen zu artikulieren (nicht bedingungslos durchzusetzen), wagen wir zum Zeitpunkt der Fertigstellung des Beitrags zu bezweifeln. Wir würden uns von einer genuin zivilmächtigen (Re-)Orientierung deutscher Namibiapolitik überzeugen lassen, sollte sich die Bundesregierung nicht nur zu einer handfesten und unverklausulierten Entschuldigung durchringen, sondern aus dieser ideellen Geste auch die entsprechenden materiellen Konsequenzen ziehen und sich unter Einbeziehung der Opfergruppen um vernünftige Formen der Wiedergutmachung - etwa in Gestalt einer Verlängerung und signifikanten Aufstockung der Sonderinitiative - bemühen.

12 Dieser pragmatische Politikansatz, das flexible und nicht das sture Verfolgen der eigenen Pläne, das Unterordnen kurzfristiger Interessen unter langfristige Ziele, das »So-weit-Entgegenkommen« wie nötig bei gleichzeitigem »So-wenig-Nachgeben « wie möglich, scheint uns eine grundlegende Handlungsregel deutscher Außenpolitik zu sein, die sich auch, aber nicht nur, in der deutschen Namibiapolitik rekonstruieren lässt (Laurer/Seidl 2014). 


\subsubsection{Vergangenheits- als Zukunftspolitik}

Es scheint uns ein zentrales Element der pragmatisch-interessenorientierten Namibiapolitik der Bundesregierung zu sein, dass diese den Blick stets in die Zukunft zu richten versucht, wenngleich sie weiß, dass sie die Vergangenheit so schnell nicht loswird (vgl. Böhlke-Itzen 2004: 98-104):

»Und es ist, sag ich aber auch, es ist Geschichte und es macht keinen Sinn sozusagen, sich in die Geschichte zu vergraben, man muss eine Erinnerungspolitik machen, das machen wir auch [...] aber wir müssen nach vorne gucken, wir müssen in die Zukunft gucken« (Repräsentant der Bundesregierung 2014).

Zukunftsorientierung ist in gewisser Weise immer auch Vergangenheitspolitik, der Kampf um das deutsche Staatswohl immer auch ein Kampf um zukünftige Möglichkeitsräume in Form eines Kampfes um mögliche Deutungen der Vergangenheit. Die langjährige deutsche Nicht-Thematisierungs-Nicht-Anerkennungs-Nicht-Bearbeitungspolitik, die sich erst jetzt und unter dem Druck der Ereignisse schrittweise aufzulösen scheint, muss daher immer auch als Schutz des deutschen Selbst- und Geschichtsbildes verstanden werden. Sie ist eine Form der Zukunftsgestaltung durch Verzicht auf angemessene Vergangenheitsbearbeitung. ${ }^{13}$ Wir gehen dabei davon aus, dass ein grundlegendes Interesse aller Regierungen der Welt darin besteht, über einen größtmöglichen (außen-)politischen Handlungsspielraum zu verfügen. Insofern es aber zutrifft, dass »Inhalt, Struktur, Umfang und Spezifik« der Wiedererzählungen von Geschichte »aktuelle Potenziale und Anschlussmöglichkeiten « (Schmid 2009: 7) und damit die Ausgestaltung politischer Handlungsräume maßgeblich prägen - wie dies Arbeiten zur Funktion des kollektiven Gedächtnisses und dessen Einfluss auf die politische Kultur und politische Möglichkeiten beschreiben (Assmann 1999; Heinrich 2009) - ist es nur naheliegend davon auszugehen, dass jede Exekutive Geschichtspolitik betreibt. Schließlich hängen die Fragen, wer wir sind und wie wir zukünftig sein wollen, maßgeblich von der Antwort ab, die wir uns selbst auf die Frage geben, wer wir waren.

Die nach 1990 geführte Diskursschlacht um die »Normalisierung« deutscher Außenpolitik, die bei genauer Betrachtung ein Kampf um die Vergrößerung außenpolitischen Handlungsspielraums - etwa in der Frage von Kampf- und out-of-area-

13 Vergangenheit wird zwar nicht ausgeblendet, steht aber gewissermaßen im Schatten einer »besseren Zukunft « und nimmt dabei eine diffuse Rechtfertigungsfunktion an. Man wünscht sich, »dass die bilateralen Beziehungen davon bestimmt sein mögen, zukunftsgerichtet die Entwicklungschancen Namibias zu erkennen und zu fördern, ohne - das möchte ich betonen - die historischen Belastungen aus der gemeinsamen Geschichte zu vernachlässigen« (Staatsminister Hoyer zit. nach Deutscher Bundestag 2011a). 
Einsätzen deutscher Streitkräfte (Roos/Rungius 2015) - war, musste vor diesem Hintergrund auch geschichtspolitisch geführt werden. ${ }^{14}$ Von der Intensität geschichtspolitischer Auseinandersetzungen zeugen nicht nur die Fischer-Kontroverse in den 1960ern oder der Historikerstreit in den 1980ern, sondern in jüngererZeit auch 1.) die Debatte um die Befunde der vom damaligen Außenminister Joschka Fischer eingesetzten Historiker-Kommission zur Erforschung politischer und personaler Kontinuitäten »im Amt « (Conze et al. 2010; Koerfer 2013), 2.) die Rezeption der von Jürgen Zimmerer ausgearbeiteten These eines historischen Zusammenhangs zwischen dem Völkermord in Südwestafrika und der Shoa ${ }^{15}$ sowie 3.) die Aktualisierung der Fischer-Kontroverse in den gegenwärtigen Bemühungen einer Umschreibung der Verantwortungsgeschichte mit Blick auf den Ausbruch des Ersten Weltkriegs. ${ }^{16}$ Diese Debatten verdeutlichen die besondere Relevanz geschichtspolitischer (Um-)Deutungen für die Ent- und Begrenzung politischer Möglichkeitsräume (Winkler 2004; Steinbach 2012). Das besondere Interesse aller Bundesregierungen an der Gestaltung dieser Debatten mündete in früheren Arbeiten in der These, »dass die

$14 »$ Wer Normalität fürchtet, weil er sich erinnert, was deutsche Normalität einmal gewesen ist, hängt an der Vergangenheit; er wird auch gegen seinen Willen zu ihrem Gefangenen. Wir müssen uns von der fast psychopathischen Last heilen, psychopathisches Leid zu kultivieren. Die Vergangenheit darf die Zukunft nicht behindern « (Bahr 2003: 137).

15 Wenngleich es durchaus gute Gründe für eine Kritik an der Zimmerer'schen Kontinuitätsthese gibt (vgl. etwa: Gerwarth/Malinowski 2007), überrascht doch die Regelmäßigkeit, mit der Zimmerer eine Gleichsetzung- oder gar Verursachungsthese unterstellt wird. Zimmerer selbst wundert sich über »die Vehemenz vieler Antworten« sowie über »die Aufgeregtheit der Debatte, das sofortige Abgleiten ins persönlich-diffamierende bei gleichzeitigem Fehlen jeglicher inhaltlicher Auseinandersetzung « (Zimmerer 2011 b: 9). Im Auswärtigen Amt selbst reagierte man bereits in den 1960er Jahren - lange vor der namibischen Unabhängigkeit oder der von den Herero angestrengten Klage allergisch auf Elemente von Kontinuität in der deutschen (Gewalt-)Geschichte insinuierende Thesen:»[W]er im Ausland aufParallelen zwischen dem Völkermord an den Hereros und den Juden und Polen hinwies, bekam es mit Zensurabsichten des Auswärtigen Amtes zu tun« (Bley 1996 b: 322). Dort sah man in derlei Behauptungen »für das deutsche Ansehen in Afrika höchst nachteilige Vorkommnisse«, die »in Zukunft durch ein engeres Zusammenarbeiten der Universitäten mit dem Auswärtigen Amt vermieden werden können« (zit. nach Böhlke-Itzen 2004: 92).

16 Für Volker Ullrich geht es dabei »um eine geschichtspolitische Weichenstellung. Was den Konservativen im »Historikerstreit« der achtziger Jahre noch missglückte - nämlich die Deutungshoheit über die eigene Geschichte zurückzugewinnen - das soll jetzt gelingen« (Ullrich 2014). »Schlafwandler, so viel steht fest, können wir nicht gebrauchen - weder in der aktuellen Politik (in einem wieder hoch explosiven Umfeld) noch in fehlerhaften Geschichtsbildern über den Ersten Weltkrieg. Denn auch der politische Nebel, den diese Metapher verbreitet, ist keineswegs harmlos, sondern potenziell gefährlich. Er ist geeignet, die deutsche Gewaltgeschichte zu glätten und zu entsorgen. Unterschwellig arbeitet dieses Bild einer Politik zu, die eine gestiegene deutsche Verantwortung in der Welt auch wieder militärisch definieren möchte. Ein von historischer Kriegsschuld gereinigtes Deutschland könnte mit Hilfe eines geglätteten Geschichtsbildes einen größeren internationalen Handlungsspielraum beanspruchen und zu einer - auch militärisch instrumentierten - neuerlichen Weltmachtpolitik verleiten« (Wette 2014: 101). 
Bundesregierung nicht dazu bereit war, aufgrund der nationalsozialistischen Vergangenheit in Zukunft auf Gestaltungsmacht zu verzichten " (Roos 2010: 186). Diese These lässt sich an dieser Stelle dergestalt ergänzen, dass die Bundesregierung ebenfalls nicht ohne Not dazu bereit ist, aufgrund der kolonialen Vergangenheit in $\mathrm{Zu}-$ kunft auf Gestaltungsmacht zu verzichten.

Vor dem Hintergrund dieser Überlegungen lässt sich weiterhin die These formulieren, dass die Bundesregierung grundsätzlich befürchtet, dass eine breite öffentliche Auseinandersetzung mit der kolonialen Vergangenheit in Deutsch-Südwestafrika erheblichen Einfluss auf die regierungsamtliche Geschichtspolitik und das öffentliche Geschichtsselbstbild in Deutschland nehmen könnte, indem sie den kolonialen Genozid aus der »Rumpelkammer des nationalen Gedächtnisses« (Kößler/ Melber 2004: 64) hervorholt und unangenehme Fragen nach einer nicht auf den Nationalsozialismus beschränkten Gewaltgeschichte deutscher Außenpolitik aufwirft. ${ }^{17} \mathrm{Zu}$ einem Zeitpunkt, da eine einflussreiche Diskurskoalition der »außenpolitischen Klasse « (Hellmann 2013: 16) die diesbezüglich weiterhin skeptische deutsche Bevölkerung auf ein »Mehr « an internationaler Verantwortungsübernahme qua Einsatz der Bundeswehr im Ausland einschwören möchte, käme eine öffentliche Debatte über das ein Jahrhundert andauernde Verschweigen der eigenen genozida-

17 Die Frage nach dem Umgang mit der kolonialen Vergangenheit lässt sich in Deutschland nicht losgelöst von der geschichtspolitisch mindestens ebenso bohrenden Frage beantworten, ob und wie die Verbrechen des Nationalsozialismus in die deutsche (Gewalt-)Geschichte einzuordnen sind. Handelt es sich bei der Shoa um ein singuläres Ereignis - und zwar nicht allein mit Blick auf das Ausmaß des Terrors und die Abgründigkeit menschlichen Seins, sondern auch im Sinne eines letztlich unerklärlichen Ausbruchs aus der eigentlich humanistischen und aufgeklärten Kultur des deutschen Nationalstaates? Oder trägt umgekehrt die ausbleibende Auseinandersetzung mit Fragen gewaltgeschichtlicher Kontinuität in der politischen Kultur des deutschen Gemeinwesens, trägt die geschichtspolitische »Enklavisierung« des Nationalsozialismus selbst zur erinnerungs- und gesellschaftspolitischen Marginalisierung des Kolonialismus bei? »Die deutsche Kolonialgeschichte ist deshalb auch unerledigt, weil sie die Erinnerung daran wecken kann, dass die industrielle Gesellschaft in Deutschland an der imperialistischen Gewalt Europas über die außereuropäische Welt mitbeteiligt war und in dieser Gesellschaft gewalttätige Traditionen vorhanden sind, die sich nicht auf den »Dämon « Hitler reduzieren lassen, sondern die sich in sozusagen »normalen« Zeiten, im Grunde in der »guten alten Zeit« vollzogen« (Bley 1996 b: 319). Es stellt sich in diesem Zusammenhang die Frage, 1.) ob eine unverblümt-unverklausulierte Anerkennung kolonialer Schuld nicht auch deswegen vermieden wird, weil damit die schmerzhafte Infragestellung eines Identitätskonzepts verbunden ist, welches eng an eine, mit Ausnahme der Jahre 1933 bis 1945, positiv konnotierte deutsche Nationalgeschichte gebunden ist (vgl. Katjavivi 2014: 156); 2.) wieso zur Aufarbeitung der kolonialen Vergangenheit (noch) nicht auf das, mit Blick auf die im internationalen Vergleich vorbildliche Aufarbeitung der nationalsozialistischen Vergangenheit in der deutschen Gesellschaft, durchaus vorhandene erinnerungspolitische Repertoire aus Gedenkpraktiken und Bearbeitungsroutinen zurückgegriffen wird; 3.) welche Rolle dabei die nachvollziehbare, letztlich aber auch nur bedingt überzeugende Angst vor einer damit möglicherweise einhergehenden Relativierung der Shoa spielt (vgl. Böhlke-Itzen 2004: 90-95; Kößler/Melber 2004: 39-40). 
len Vergangenheit der deutschen Schutztruppe aus Perspektive der Bundesregierung zur Unzeit. ${ }^{18}$ Unsere These lautet daher, dass in einer Situation, die davon geprägt ist, die militärische Zurückhaltung der deutschen Gesellschaft immer weiter aufzulösen, um außenpolitischen Handlungsspielraum zu gewinnen, eine geschichtspolitische Debatte über einen bislang weitgehend ignorierten Völkermord nicht im Interesse der Bundesregierung liegt und wenn nur irgendwie möglich verhindert werden soll. Neben dem Ziel der Vermeidung von Reparationszahlungen wird die Strategie der Bundesregierung also auch von geschichtspolitischen Erwägungen geleitet und durch eine pragmatische Taktik des (Ver-)Schweigens und DeThematisierens - in der freien Wirtschaft spricht man von silencing (Thiesmeyer 2003) - flankiert.

Die jüngst erfolgte Neupositionierung der Bundesregierung in der Völkermordfrage stellt aber auch vor diesem Hintergrund eher einen adaptiven Strategie- als einen radikalen Kurswechsel dar. Schließlich konterkarierte die Politisierung des Themas letztlich die vergangenheitspolitischen Ziele der regierungsamtlichen Begriffsvermeidungsstrategie, indem das in dieser angelegte Bigotteriepotenzial zum politisch-moralischen Zündstoff einer öffentlich-medialen Aufmerksamkeitsspirale wurde. Neuere Paradigmen der Unternehmenskommunikation setzen in solchen Situationen nicht mehr auf silencing, sondern auf Transparenz und klare Verantwortungsübernahme. Analog dazu lag und liegt es auch aus Perspektive der Bundesregierung nahe, das Thema nicht durch eine fortgesetzte Verweigerungshaltung immer größer werden zu lassen, sondern kontrolliert Luft aus dem Aufmerksamkeitsballon zu lassen. Dass dies nicht im Rahmen einer symbolischen Geste, sondern in geradezu erstaunlicher zeremonieller Lakonie geschah, deutet darauf hin, dass der Bundesregierung weiterhin an einer möglichst geringen medialen Präsenz und einer möglichst geräuschlosen öffentlichen Bearbeitung des Themas gelegen ist. Was sich geändert hat, ist, dass eine für die regierungsamtliche Geschichtspolitik potenziell schädliche - weil gegenwärtige und zukünftige Möglichkeitsräume verengende -, breitere gesellschaftliche Auseinandersetzung mit der kolonialen Vergangenheit in Zukunft nicht mehr durch rigorose Begriffsvermeidung, sondern durch (juristisch unverfängliches) begriffliches Entgegenkommen zu verhindern versucht wird.

18 So etwa der amtierende Bundespräsident, der - um seinen Aufruf zu größerer weltpolitischer Verantwortungsübernahme zu begründen - zunächst einmal sagen muss: »Dies ist ein gutes Deutschland « (Gauck 2014). 


\section{Eine andere Interessenpolitik?}

1959 stellte Hasso von Etzdorf, damals Leiter der Länderabteilung III des Auswärtigen Amtes, selbstbewusst fest, dass Deutschland als »kolonial unbelastetem und wirtschaftlich hochentwickeltem Land « eine »natürliche Vermittlerrolle« zwischen Afrika und den bisherigen Kolonialmächten zufalle: Wir verfügen über ein großes Plus in Afrika - den Verlust unserer Kolonien vor vierzig Jahren « (zit. nach Krüger 2003: 132). Dass eine solche Einschätzung nicht nur »der Zeit« und möglicherweise politischer Gesinnung geschuldet war, wird deutlich, wenn wir den inzwischen eines Besseren belehrten GRÜNEN-Parlamentarier Hans-Christian Ströbele (vgl. Deutscher Bundestag 2004) 40 Jahre später sagen hören,

»dass Deutschland das Glück hatte, sehr früh aus der Kolonialisierung gewaltsam herausgetrieben worden zu sein. [...] Dies ist eine Chance [...]. Deutschland kann eine Rolle übernehmen, die unbelastet ist und die deshalb eine Vorreiterrolle sein kann« (zit. nach Kößler/Melber 2004: 37).

Nun hat sich die Hoffnung, die vergleichsweise »kurze« deutsche Kolonialgeschichte könnte als Legitimationsgrundlage einer »natürlichen Vermittlerrolle« politisch urbar gemacht werden, schon früh als unbegründet herausgestellt (Bley 1996 b: 317). Wir möchten zum Abschluss drei weniger politikwissenschaftlich, denn politikberatend motivierte Argumente formulieren, weshalb die Vorstellung einer unbelasteten Kolonialgeschichte und daraus gespeiste politische Hoffnungen im Zeitalter von Globalisierung und new international morality (Schaller 2011:267) nicht mehr nur normativ problematisch, aber politisch irrelevant sind, sondern gerade aufgrund ihres normativen Problemcharakters zu einem politischen Problem werden (können).

1.) Zunächst steht die auch nach den jüngsten Ereignissen fortdauernde Marginalisierung des Kolonialismus in Deutschland in einem immer öfter aufbrechenden Spannungsverhältnis zur konstitutiven Rolle, welche die deutsche Kolonialherrschaft für das Selbstverständnis der betroffenen Bevölkerungsgruppen in Namibia hat. Dass Roman Herzog 1998 die »Periode« des deutschen Kolonialismus »mit dem Ende des ersten Weltkrieges« für »abgeschlossen« erklärte (zit. nach Krüger 2003: 124), ist ja nicht nur mit Blick auf die Nachwirkungen des Kolonialismus in Deutschland selbst eine zutiefst fragwürdige Aussage (Eckert/Wirz 2002; Ha 2005); sie ist es auch mit Blick auf die Präsenz des Kolonialen in der namibischen Gegenwartsgesellschaft. Ob es will oder nicht, Deutschland ist spätestens mit den Anerkennungs- und Reparationsforderungen der Opfergruppen zu einer ehemaligen Kolonialmacht »geworden« (vgl. Krüger 2003). Eine den Völkermord ausklammernde 
Vergangenheitspolitik, die darauf spekuliert, dass die gemeinsame Kolonialgeschichte für die Beziehungen zwischen Deutschland und Namibia im Laufe der Zeit irrelevant wird, war daher von Beginn an verfehlt und ist es auch weiterhin. Es gibt auf der anderen Seite keinen Grund anzunehmen, dass die deutsch-namibischen Beziehungen unter einer offiziellen und unverstellten Entschuldigung von prominenter Stelle Schaden nehmen könnten. ${ }^{19}$ Die Bundesregierung wäre also gut beraten, ihren zum Zeitpunkt der Finalisierung des Forschungsberichts eingeschlagenen Weg fortzusetzen und die jüngste Anerkennung des Völkermords zum Auftakt eines umfassenden, die Opfergruppen einschließenden Versöhnungsdialogs zu machen - und diese nicht wie die Rede Heidemarie Wieczorek-Zeuls zur Ausnahme werden zu lassen.

2.) Wenn Deutschland eine »führende« Rolle bei der Gestaltung der Welt einnehmen möchte, dann ist es heute mehr denn je - so behaupten wir - auf die »Ressource« Glaubwürdigkeit angewiesen. Glaubwürdigkeit sichert als zentrale politische »Ressource« der Gegenwart die Autorität und Legitimität von Führung. Wer nun in Zeiten, in denen es eine wachsende internationale Erwartungshaltung hinsichtlich der Anerkennung und Wiedergutmachung vergangener Verbrechen gibt (Schaller 2011: 267), glaubwürdig sein und damit führen will, der muss sich seiner Geschichte in moralisch angemessener Weise stellen (Howard-Hassmann 2008: 16-18). Die Aussöhnung mit dem globalen Süden ist dabei in Zeiten des relativen Bedeutungsverlusts des Westens, in einem Moment des Übergangs von einer Position relativer Stärke zu einer Position relativer Schwäche, nicht nur geopolitisch klug, sondern gehört auch zu den bedeutsamsten Zukunftsaufgaben einer zivilmächtigen Weltfriedenspolitik. ${ }^{20}$ Hier könnte Deutschland Vorbild sein und »führen«, hier könnte es eine Vorreiter- oder gar Vermittlerrolle einnehmen, hier könnte es »Verantwortung übernehmen « und diese Rolle ohne Euphemismus-Verdacht mit Leben füllen. Der deutsche Name und damit auch der deutsche Einfluss in der Welt

19 »Eine Entschuldigung der Bundeskanzlerin, des Außenministers, des Bundespräsidenten, das wäre eine vertrauensbildende Maßnahme. Und ich denke nicht, dass die namibische Regierung dagegen wäre, wenn man sich bei den Opfergruppen entschuldigt - überhaupt nicht. Das waren ja schließlich die, die am meisten gelitten haben « (Wietersheim 2014).

20 Dabei geht die langjährige und zum Zeitpunkt der Finalisierung des Beitrags noch immer geltende Sprachregelung - wir können »We feel sorry«, aber nicht »We apologize« sagen (Repräsentant Bundesregierung 2014) - völlig an der Erkenntnis vorbei, dass für »eine gleichberechtigte Zusammenarbeit und für ein erstarkendes afrikanisches Selbstbewusstsein [...] das offene Eingestehen von Fehlern wichtig und unverzichtbar « ist (Anke Eymer (CDU) zit. nach Deutscher Bundestag 2008). Bedauern kann dabei ebenso wenig für eine ausbleibende Entschuldigung entschädigen, wie der instrumentelle Gebrauch von Entwicklungshilfe das Ausbleiben einer - zumindest symbolisch als solcher gerahmten - Entschädigung entschuldigt (Howard-Hassmann 2008: 4; Sarkin 2009: 192). 
würden darunter jedenfalls nicht leiden. Wer sich dagegen weigert, das Unrecht von gestern ein- und dafür geradezustehen, darf kaum erwarten, dass ihm vorbehaltlos geglaubt wird, wenn er mit Blick auf die Krisen und Verbrechen der Gegenwart selbstlos Verantwortung zu übernehmen verspricht.

3.) Glaubwürdig sollte deutsche Außenpolitik schließlich aber auch in Bezug auf den Verfassungsauftrag des Grundgesetzes sein. Nicht nur, dass es im Sinne des Grundgesetzes nur als problematisch gelten kann, dass die Bundesregierung durch ihre jahrzehntelange Verweigerungshaltung in der Völkermordfrage Öl ins Feuer kolonialapologetischer Geisteshaltungen gegossen hat (vgl. Kößler/Melber 2004: 64). Die bundesdeutsche Haltung in der Völkermord- und Reparationsfrage ist auch deswegen problematisch, weil sie mitunter noch immer zu verkennen scheint, dass es sich dabei um »keine legale Frage [handelt], sondern primär [um] eine der politischen Ethik beziehungsweise der praktischen Solidarität (Engert 2009: 294). Als solche ist sie im 21. Jahrhundert aber auch eine Frage einer angemessenen Weltfriedenspolitik einer glaubwürdigen Zivilmacht und nicht weniger als dies haben wir uns selbst in der Präambel unseres Grundgesetzes versprochen.

\section{Literatur}

Anderson, Rachel J. 2005: Redressing Colonial Genocide. The Hereros' Cause of Action Against Germany, in: California Law Review 93, 1055-1090.

Assmann, Aleida 1999: Erinnerungsräume: Funktionen und Wandlungen des kulturellen Gedächtnisses, München.

Bahr, Egon 2003: Der deutsche Weg. Selbstverständlich und normal, München.

Baumann, Rainer 2006: Der Wandel des deutschen Multilateralismus, Baden- Baden.

Bley, Helmut 1996 a: Namibia, die Bundesrepublik und der Westen: 15 Jahre Krisenverschärfung, in: ders. (Hrsg.): Afrika, Geschichte und Politik. Ausgewählte Beiträge 1967-1992, Berlin, 201-223.

Bley, Helmut 1996 b: Unerledigte deutsche Kolonialgeschichte, in: ders. (Hrsg.): Afrika, Geschichte und Politik. Ausgewählte Beiträge 1967-1992, Berlin, 317-323.

Böhlke-Itzen, Janntje 2004: Kolonialschuld und Entschädigung. Der deutsche Völkermord an den Hereros (1904-1907), Frankfurt a. M.

Brehl, Medardus 2007: Vernichtung der Herero. Diskurse der Gewalt in der deutschen Kolonialliteratur, München.

Brenke, Gabriele 1989: Die Bundesrepublik Deutschland und der Namibia-Konflikt, München. 
Bundesregierung 2015: Regierungspressekonferenz vom 10.7.2015, in: http:// www.bundesregierung.de/Content/DE/Mitschrift/Pressekonferenzen/2015/07/ 2015-07-10-regpk.html; 31.7.2015.

CIA 2013: The CIA world factbook 2014, New York.

Conrad, Sebastian 2008: Deutsche Kolonialgeschichte, München.

Conze, Eckart/Frei, Norbert/Hayes, Peter/Zimmermann, Moshe 2010: Das Amt und die Vergangenheit. Deutsche Diplomaten im Dritten Reich und in der Bundesrepublik, München.

Deutscher Bundestag 1989 a: Stenographischer Bericht der 129. Sitzung der 11. Wahlperiode (Plenarprotokoll 11/129, 24.2.1989), in: http://dipbt.bundestag.de/ doc/btp/11/11129.pdf, 31.8.2015.

Deutscher Bundestag 1989 b: Beschlußempfehlung und Bericht des Auswärtigen Ausschusses (Drucksache 11/4205, 15.3.1989) in: http://dip21.bundestag.de/ dip21/btd/11/042/1104205.pdf, 31.8.2015 .

Deutscher Bundestag 2004: Stenographischer Bericht der 114. Sitzung der 15. Wahlperiode (Plenarprotokoll 15/144, 17.6.2004), in: http://dip21.bundestag.de/ dip21/btp/15/15114.pdf, 31.8.2015.

Deutscher Bundestag 2008: Stenographischer Bericht der 172. Sitzung der 16. Wahlperiode (Plenarprotokoll 16/172, 26.6.2008), in: http://dip21.bundestag.de/ dip21/btp/16/16172.pdf, 31.8.2015.

Deutscher Bundestag 2011 a: Stenographischer Bericht der 145. Sitzung der 17. Wahlperiode (Plenarprotokoll 17/145, 30.11.2011), in: http://dipbt.bundestag. de/doc/btp/17/17145.pdf, 31.8.2015.

Deutscher Bundestag 2011 b: Antwort der Bundesregierung (Drucksache 17/8057, 1.12.2011), in: http://genocide-namibia.net/wp-content/uploads/2015/01/17080 57_Antwort.pdf, 31.8.2015.

Deutscher Bundestag 2012 a: Antrag der Fraktionen SPD und BÜNDNIS 90/DIE GRÜNEN (Drucksache 17/9033 (neu), 20.3.2012), in: http://dip21.bundestag.de/dip21/btd/17/090/1709033.pdf, 31.8.2015.

Deutscher Bundestag 2012 b:. Stenographischer Bericht der 168. Sitzung der 17. Wahlperiode (Plenarprotokoll 17/168, 22.3.2012), in: http://dipbt.bundestag.de/ doc/btp/17/17168.pdf, 31.8.2015.

Deutscher Bundestag 2012 c: Antwort der Bundesregierung (Drucksache 17/9255, 3.4.2012), in: http://genocide-namibia.net/wp-content/uploads/2015/01/170925 5_Antwort-Kleine-Anfrage.pdf, 31.8.2015.

Deutscher Bundestag 2012 d: Antwort der Bundesregierung (Drucksache 17/10481, 14.8.2012), in: http://genocide-namibia.net/wp-content/uploads/2015/01/17104 81_Antwort.pdf, 31.8.2015. 
Deutscher Bundestag 2013: Schriftliche Fragen mit den in der Woche vom 18. November 2013 eingegangenen Antworten der Bundesregierung (Drucksache 18/82, 18.11.2013), in: http://dip21.bundestag.de/dip21/btd/18/000/180008 2.pdf, 31.8.2015.

Deutscher Bundestag 2015: Antwort der Bundesregierung (Drucksache 18/5166, 12.6.2015), in: http://dip21.bundestag.de/dip21/btd/18/051/1805166.pdf, 31.8.2015.

Dewey, John 1991: The Public and its Problems, Athens, OH.

Drechsler, Horst 1966: Südwestafrika unter deutscher Kolonialherrschaft. Der Kampf der Herero und Nama gegen den deutschen Kolonialismus (1884-1915), Berlin.

Du Pisani, André 2010: The Discursive Limits of SWAPO's Dominant Discourses on Anti-Colonial Nationalism in Postcolonial Namibia. A First Exploration, in: Du Pisani, André/Kößler, Reinhart/Lindeke, William A. (Hrsg.): The Long Aftermath of War. Reconciliation and Transition in Namibia, Freiburg, 1-40.

Eckert, Andreas/Wirz, Albert 2002: Wir nicht, die anderen auch. Deutschland und der Kolonialismus, in: Conrad, Sebastian/Randeria, Shalini (Hrsg.): Jenseits des Eurozentrismus. Postkoloniale Perspektiven in den Geschichts- und Kulturwissenschaften, Frankfurt a. M., 372-392.

Eicker, Steffen 2009: Der Deutsch-Herero-Krieg und das Völkerrecht. Die völkerrechtliche Haftung der Bundesrepublik Deutschland für das Vorgehen des Deutschen Reiches gegen die Herero in Deutsch-Südwestafrika im Jahre 1904 und ihre Durchsetzung vor einem nationalen Gericht, Frankfurt a. M.

Engert, Stefan 2009: Politische Schuld, moralische Außenpolitik? Deutschland Namibia und der lange Schatten der kolonialen Vergangenheit, in: Harnisch, Sebastian/Maull, Hanns W./Schieder, Siegfried (Hrsg.): Solidarität und internationale Gemeinschaftsbildung. Beiträge zur Soziologie der internationalen Beziehungen, Frankfurt a. M., 277-303.

Franke, Ulrich/Roos, Ulrich 2010: Actor, Structure, Process. Transcending the State Personhood Debate by Means of a Pragmatist Ontological Model for International Relations Theory, in: Review of International Studies 36: 4, 1057-1077.

Franke, Ulrich/Roos, Ulrich (Hrsg.) 2013: Rekonstruktive Methoden der Weltpolitikforschung. Anwendungsbeispiele und Entwicklungstendenzen, Baden- Baden.

Ganns, Harald 2002: Der Anfang war schwer. Die ersten Jahre der deutschen diplomatischen Präsenz in Namibia, in: Hess, Klaus/Becker, Klaus (Hrsg.): Vom Schutzgebiet bis Namibia 2000, Göttingen, 85-92. 
Gauck, Joachim 2014: Deutschlands Rolle in der Welt: Anmerkungen zu Verantwortung, Normen und Bündnissen (Rede anlässlich der Eröffnung der »Münchner Sicherheitskonferenz«, 31.1.2014), München.

Geingob, Hage 2014: Prime Minister Dr. Hage G. Geingob's Parliamentary Statement on the Issue of Genocide and Reparations, and the Rise of Tribalism (New Era, 6.8.2014), in: http://www.newera.com.na/2014/08/06/prime/; 31.7.2015.

Gerwarth, Robert/Malinowski, Stefan 2007: Der Holocaust als »kolonialer Genozid«? Europäische Kolonialgewalt und nationalsozialistischer Vernichtungskrieg, in: Geschichte und Gesellschaft 33, 439-466.

Gewald, Jan-Bart 1996: Towards Redemption. A Socio-Political History of the Herero of Namibia between 1890 and 1923, Leiden.

Gewald, Jan-Bart 2005: Das deutsche Kaiserreich und die Herero des südlichen Afrika. Der Völkermord und die Entschädigungsfrage, in: Jones, Adam (Hrsg.): Völkermord, Kriegsverbrechen und der Westen, Berlin, 69-90.

Grill, Bartholomäus 2004: Aufräumen, aufhängen, niederknallen! (Die Zeit, 5.8.2004), in: http://www.zeit.de/2004/33/Herero-Kurzfassung/komplettansicht; 31.7.2015.

Ha, Kien Nghi 2005: Mach(t)raum(a) Berlin. Deutschland als Kolonialgesellschaft, in: Eggers, Maureen Maisha/Kilomba, Grada/Pesche, Peggy/Arndt, Susan (Hrsg.): Mythen, Masken und Subjekte. Kritische Weissseinsforschung in Deutschland, Münster, 105-117.

Harring, Sidney L. 2007: The Herero Demand for Reparation from Germany. The Hundred Year Old Legacy of a Colonial War in the Politics of Modern Namibia, in: Du Plessis, Max/Stephen, Peté (Hrsg.): Repairing the Past? International Perspectives on Reparations for Gross Human Rights Abuse, Antwerpen, 437-450.

Haspel, Annelie 1995: Kontinuität oder Wandel? Das besondere Verhältnis von Deutschen zu Namibia, Hamburg.

Heinrich, Horst-Alfred 2009: Kollektive Erinnerung im System, in: Schmid, Harald (Hrsg.): Geschichtspolitik und kollektives Gedächtnis. Erinnerungskulturen in Theorie und Praxis, Göttingen, 77-91.

Hellmann, Gunther 2013: Reflexive Sicherheitspolitik als antihegemoniales Rezept.

Deutschlands Sicherheit und seine gewandelte Strategie, Beitrag mit Anmerkungen, in: http://www.fb03.uni-frankfurt.de/47830385/ APUZ_2013_Anm_www.pdf?; 11.8.2015.

Hellmann, Gunther/Weber, Christian/Sauer, Frank 2008: Die Semantik der neuen deutschen Außenpolitik. Eine Analyse des außenpolitischen Vokabulars seit Mitte der 1980er Jahre, Wiesbaden. 
Howard-Hassmann, Rhoda E. 2008: Reparations to Africa, mit Lombardo, Anthony P., Philadelphia, PA.

Jaguttis, Malte 2010: Paths to a Hearing of the Herero Case Under International Law. Beyond the Patterns of Colonial Self-Description?, in: Schmidt, Dierk (Hrsg.): The Division of the Earth. Tableaux on the Legal Synopses of the Berlin Africa Conference, Köln, 76-84.

Jamfa, Leonard 2008: Germany Faces Colonial History in Namibia. A Very Ambiguous "I Am Sorry", in: Gibney, Mark (Hrsg.): The Age of Apology. Facing up to the Past. Philadelphia, PA, 202-215.

Johnson, Dominic 2015: Nur der erste Schritt, in: taz, 10.7.2015, in: http:// www.taz.de/!5212170/; 31.7.2015.

Kämmerer, Jörn Axel/Föh, Jörg 2004: Das Völkerrecht als Instrument der Wiedergutmachung? Eine kritische Betrachtung am Beispiel des Herero-Aufstandes, in: Archiv des Völkerrechts 42, 294-328.

Katjavivi, Peter H. 2014: Namibia's Bilateral Relations with Germany. A Crucial Relationship, in: Bösl, Anton/Du Pisani, André/Zaire, Dennis U. (Hrsg.): Namibia's Foreign Relations. Historic Contexts, Current Dimensions, and Perspectives for the 21st Century, Windhoek, 135-168.

Kauraisa, Jesse Jackson 2015: Germany Trivialises Genocide Claims (Windhoek Observer, 17.7.2015), in: http://observer24.com.na/8-latest-news/4816-germany-trivialises-genocide-claims; 31.7.2015.

Koerfer, Daniel 2013: Diplomatenjagd. Joschka Fischer, seine Unabhängige Historikerkommission und Das AMT, Potsdam.

Kößler, Reinhart 2008: Entangled History and Politics. Negotiating the Past Between Namibia and Germany, in: Journal of Contemporary African Studies 26: 3, 313-339.

Kößler, Reinhart 2011: Zweierlei Amnesie und die komplexe postkoloniale Lage Namibias, in: Friedenswarte 86: 1-2, 73-99.

Kößler, Reinhart; Melber, Henning 2004: Völkermord und Gedenken. Der Genozid an den Herero und Nama in Deutsch-Südwestafrika 1904-1908, in: Wojak, Irmtrud/Meinl, Susanne (Hrsg.): Völkermord und Kriegsverbrechen in der ersten Hälfte des 20. Jahrhunderts. Frankfurt a. M., 37-75.

Krüger, Gesine 1999: Kriegsbewältigung und Geschichtsbewusstsein. Realität, Deutung und Verarbeitung des deutschen Kolonialkriegs in Namibia 1904 bis 1907, Göttingen. 
Krüger, Gesine 2003: Vergessene Kriege. Warum gingen die deutschen Kolonialkriege nicht in das historische Gedächtnis der Deutschen ein?, in: Buschmann, Nikolaus/Langewiesche, Dieter (Hrsg.): Der Krieg in den Gründungsmythen europäischer Nationen und der USA, Frankfurt a. M., 120-137.

Lammert, Norbert 2015: Deutsche ohne Gnade (Die Zeit, 9.7.2015), in: http:// www.zeit.de/2015/28/voelkermord-armenier-herero-nama-norbert-lammert/ komplettansicht; 11.8.2015.

Laurer, Moritz/Seidl, Timo 2014: Das doppelte Ziel der deutschen Eurokrisenpolitik, in: WeltTrends 99, 120-126.

Madley, Benjamin 2004: Patterns of Frontier Genocide 1803-1910. The Aboriginal Tasmanians, the Yuki of California, and the Herero of Namibia, in: Journal of Genocide Research 6: 2, 167-192.

Maull, Hanns W. 1992: Zivilmacht Bundesrepublik Deutschland. Vierzehn Thesen für eine neue deutsche Außenpolitik, in: Europa-Archiv 10/1992, 269-278.

Maull, Hanns W. 2011: Deutsche Außenpolitik. Orientierungslos, in: Zeitschrift für Politikwissenschaft 21: 1, 93-117.

Melber, Henning 1987: Bonns Namibia-Politik. Resolution 435, Kontaktgruppe und besondere Verantwortung. Eine kritische Bestandsaufnahme, in: Vereinte Nationen 35: 2, 45-50.

Messner, Dirk 2007: Entwicklungspolitik als globale Strukturpolitik, in: Jäger, Thomas (Hrsg.): Deutsche Außenpolitik. Sicherheit, Wohlfahrt, Institutionen und Normen, Wiesbaden, 414-442.

Nuscheler, Franz 2007: Entwicklungspolitik, in: Schmidt, Siegmar/Hellmann, Gunther/Wolf Reinhard (Hrsg.): Handbuch zur deutschen Außenpolitik, Wiesbaden, 672-683.

Oevermann, Ulrich 1991: Genetischer Strukturalismus und das sozialwissenschaftliche Problem der Erklärung der Entstehung des Neuen, in: Müller-Doohm, Stefan (Hrsg.): Jenseits der Utopie. Theoriekritik der Gegenwart, Frankfurt a. M., 267-336.

Olusoga, David/Erichsen, Casper W. 2010: The Kaiser's Holocaust. Germany's Forgotten Genocide and the Colonial Roots of Nazism, London.

Pieper, Cornelia 2011: Ansprache von Staatsministerin Pieper anlässlich der Feierstunde zur Übergabe von Schädeln namibischen Ursprungs in der Charité (30.9.2011), in: http://www.auswaertiges-amt.de/DE/Infoservice/Presse/Reden/2011/110930-StMPieper-Rede-HereroNama.html; 31.8.2015.

Peirce, Charles Sanders 1998: What Pragmatism Is, in: The Peirce Edition Project (Hrsg.): The Essential Peirce. Selected Philosophical Writings Vol. 2 (1893-1913), Bloomington, IN, 331-345. 
Repräsentant der Bundesregierung 2014: Interview mit den Autoren, Windhuk, 16.9.2014.

Roos, Ulrich 2010: Deutsche Außenpolitik. Eine Rekonstruktion der grundlegenden Handlungsregeln, Wiesbaden.

Roos, Ulrich 2012: Deutsche Außenpolitik nach der Vereinigung. Zwischen ernüchtertem Idealismus und realpolitischem Weltordnungsstreben, in: Zeitschrift für Internationale Beziehungen 19: 2, 7-40.

Roos, Ulrich 2013: Grounded Theory als Instrument der Weltpolitikforschung. Die Rekonstruktion außenpolitischer Kultur als Beispiel, in: Franke, Ulrich/Roos, Ulrich (Hrsg.): Rekonstruktive Methoden der Weltpolitikforschung. Anwendungsbeispiele und Entwicklungstendenzen, Baden-Baden, 309-348.

Roos, Ulrich 2015: Beliefs and Loyalties in World Politics. A Pragmatist Framework of Analysis, in: Hellmann, Gunther/Jørgensen, Knud Erik (Hrsg.): Theorizing Foreign Policy in a Globalized World, Basingstoke, 176-198.

Roos, Ulrich/Rungius, Charlotte 2015: Neue Macht, neue Mächte - gute Gründe? Deutsche Außenpolitik und die Diskursoffensive deutscher Realpolitiker, unv. Ms.

Sarkin, Jeremy 2009: Colonial Genocide and Reparations Claims in the 21st Century. The Socio-Legal Context of Claims under International Law by the Herero Against Germany for Genocide in Namibia. 1904-1908, Westport, CT.

Sasman, Catherine 2011: Namibia Supports Genocide Claims (The Namibian, 3.11.2011), in: http://www.namibian.com.na/indexx.php?archive_id=87667\&page_type=archive_story_detail\&page $=1321 ; 31.7 .2015$.

Schaller, Dominik J. 2011: The Struggle for Genocidal Exclusivity. The Perception of the Murder of the Namibian Herero (1904-1908) in the Age of New International Morality, in: Perraudin, Michael/Zimmerer, Jürgen (Hrsg.): German Colonialism and National Identity, New York, NY, 265-277.

Schmid, Harald 2009: Geschichte, Erinnerung, Politik. Einführende Überlegungen, in: ders. (Hrsg.): Geschichtspolitik und kollektives Gedächtnis. Erinnerungskulturen in Theorie und Praxis, Göttingen, 7-20.

Schmidt-Lauber, Brigitta 2000: »Liebe Landsleute«. Deutsche in Namibia und die Bande zwischen den Kontinenten. In: Rainer Alsheimer, Alois Moosmüller und Klaus Roth (Hrsg.): Lokale Kulturen in einer globalisierenden Welt. Perspektiven auf interkulturelle Spannungsfelder. Münster: Waxmann, 243-254.

Speitkamp, Winfried 2014: Deutsche Kolonialgeschichte, 3. Auflage, Ditzingen. Steinbach, Peter 2012: Geschichte im politischen Kampf, Bonn.

Strauss, Anselm 1987: Qualitative Analysis for Social Scientists, Cambridge. 
Strauss, Anselm/Corbin, Juliet 1998: Basics of Qualitative Research. Techniques and Procedures for Developing Grounded Theory, London.

Strübing, Jörg 2005: Pragmatistische Wissenschafts- und Technikforschung. Theorie und Methode, Frankfurt a. M.

Thiesmeyer, Lynn Janet 2003: Discourse and Silencing. Representation and the Language of Displacement, Amsterdam.

Trotha, Trutz von 2003: Genozidaler Pazifizierungskrieg. Soziologische Anmerkungen zum Konzept des Genozids am Beispiel des Kolonialkrieges in DeutschSüdwestafrika 1904-1907, in: Zeitschrift für Genozidforschung 2, 31-58.

Ullrich, Volker 2014: Nun schlittern sie wieder (Die Zeit, 24.1.2014), in: http:// www.zeit.de/2014/04/erster-weltkrieg-clark-fischer/komplettansicht;

31.7.2015.

Verheugen, Günter 1986: Apartheid. Südafrika und die deutschen Interessen am Kap, Köln.

Wallace, Marion 2011: A History of Namibia. From the Beginning to 1990, London. Wette, Wolfram 2014: Seit hundert Jahren umkämpft. Die Kriegsschuldfrage, in:

Blätter für deutsche und internationale Politik 9/2014, 91-101.

Wieczorek-Zeul, Heidemarie 2007: Welt bewegen. Erfahrungen und Begegnungen, Berlin.

Wietersheim, Anton von 2014: Interview mit den Autoren, Windhuk, 12.9.2014. Winkler, Heinrich August 2004: Griff nach der Deutungsmacht. Zur Geschichte der Geschichtspolitik in Deutschland, Göttingen.

Woeller, Axel 2005: Die Landfrage und Landreform in Namibia, München.

Zeller, Joachim 2011: Ombepera i koza - Die Kälte tötet mich. Zur Geschichte des Konzentrationslagers in Swakopmund (1904-1908), in: Zimmerer, Jürgen/ Zeller, Joachim (Hrsg.): Völkermord in Deutsch-Südwestafrika. Der Kolonialkrieg (1904-1908) in Namibia und seine Folgen, Augsburg, 64-79.

Zimmerer, Jürgen 2001: Deutsche Herrschaft über Afrikaner. Staatlicher Machtanspruch und Wirklichkeit im kolonialen Namibia, Münster.

Zimmerer, Jürgen 2005: Rassenkrieg und Völkermord. Der Kolonialkrieg in Deutsch-Südwestafrika und die Globalgeschichte des Genozids, in: Melber, Henning (Hrsg.): Genozid und Gedenken. Namibisch-deutsche Geschichte und Gegenwart, Frankfurt a. M., 23-48.

Zimmerer, Jürgen (Hrsg.) 2011 a: Von Windhuk nach Auschwitz? Beiträge zum Verhältnis von Kolonialismus und Holocaust, Münster.

Zimmerer, Jürgen 2011 b: Vorwort, in: ders. (Hrsg.): Von Windhuk nach Auschwitz? Beiträge zum Verhältnis von Kolonialismus und Holocaust, Münster, 9-11. 
Zimmerer, Jürgen 2011 c: Der Wahn der Planbarkeit. Unfreie Arbeit, Vertreibung, und Völkermord als Elemente der Bevölkerungsökonomie in Deutsch-Südwestafrika, in: ders. (Hrsg.): Von Windhuk nach Auschwitz? Beiträge zum Verhältnis von Kolonialismus und Holocaust, Münster, 71-91.

\section{Die Autoren}

Dr. Ulrich Roos ist Akademischer Rat am Lehrstuhl für Politikwissenschaft, Friedens- und Konfliktforschung der Universität Augsburg.

E-Mail: ulrich.roos@phil.uni-augsburg.de

Timo Seidl ist Studierender der Soziologie an der Goethe-Universität Frankfurt am Main.

E-Mail: timo.seidl@outlook.com 